\title{
Low-thrust Trajectory Design using Closed-loop Feedback-driven Control Laws and State-dependent Parameters
}

\author{
Harry Holt * \\ Surrey Space Centre, University of Surrey, GU2 7XH, Guildford, United Kingdom \\ Roberto Armellin ${ }^{\dagger}$ \\ Department of Aerospace Vehicles Design and Control, Institut Supérieur de L'Aéronautique et de l'Espace, 31055, \\ Toulouse, France \\ Andrea Scorsoglio $\$$ and Roberto Furfaro ${ }^{\S}$ \\ Department of Systems and Industrial Engineering, University of Arizona, USA
}

\begin{abstract}
Low-thrust many-revolution trajectory design and orbit transfers are becoming increasingly important with the development of high specific impulse, low-thrust engines. Closed-loop feedback-driven (CLFD) control laws can be used to solve these trajectory design problems with minimal computational cost and offer potential for autonomous guidance. However, they have user-defined parameters which limit their optimality. In this work, an actor-critic reinforcement learning framework is proposed to make the parameters of the Lyapunov-based Q-law state-dependent, ensuring the controller can adapt as the dynamics evolve during a transfer. The proposed framework should be independent of the particular CLFD control law and provides improved solutions for mission analysis. There is also potential for future on-board autonomous use, as trajectories are closed-form and can be generated without an initial guess. The current results focus on GTO-GEO transfers in Keplerian dynamics and later with eclipse and $J_{2}$ effects. Both time-optimal and mass-optimal transfers are presented, and the stability to uncertainties in orbit determination are discussed. The task of handling orbit perturbations is left to future work.
\end{abstract}

\section{Introduction}

Low-thrust many-revolution trajectory design and orbit transfer are becoming increasingly important with the development of high specific impulse, low-thrust engines such as electric propulsion (EP) systems. The near continuous thrust has resulted in the need for trajectory design techniques that no longer approximate manoeuvres as impulsive. This increase in computational difficultly due to the required thrust arc length has impacts for both mission design phases and, perhaps more importantly, future on-board automatic guidance.

Conventional direct and indirect optimisation methods for computing low-thrust transfers are available and can solve complex optimal control problems [1, 2]. However, they are computationally intensive and still present many challenges. Direct methods generate a large optimisation problem and cannot produce the exact optimal solutions due to limitations in the control parameterisation. However, they can handle a variety of dynamics (e.g. perturbations) and constraints (e.g. eclipses). Indirect methods can produce rigorously optimal solutions but are intensive due to the need for continuation techniques to solve many-revolutions, and get complex when constraints are included. Both methods provide point solutions, i.e. for the assumed initial condition, and cannot be used as guidance laws due to time limitations and difficulties in ensuring convergence [3].

The emergence of heuristic control methods, particularly those involving closed-loop feedback-driven (CLFD) control, has allowed the computation of sub-optimal trajectories with minimal computational cost [4, 5]. They consider the trajectory design problem from a targeting perspective rather than an optimisation one, meaning they are often sub-optimal. CLFD control laws only require knowledge of the current spacecraft state and the target state in order to determine the control to be applied. This makes them suitable as initial guesses for indirect and direct methods, as in

\footnotetext{
*PhD student, Surrey Space Centre, BA building, University of Surrey, GU2 7XH, Guildford, United Kingdom. Email: h.holt@ surrey.ac.uk

† Professor, Department of Aerospace Vehicles Design and Control, Institut Supérieur de L'Aéronautique et de 1'Espace, 31055, Toulouse, France ${ }^{\ddagger}$ PhD student, System Industrial Engineering Department, University of Arizona, 1127 E. James E. Rogers Way, Tucson, AZ 85721

§ Professor, System Industrial Engineering Department, University of Arizona, 1127 E. James E. Rogers Way, Tucson, AZ 85721
} 
[6, 7], and they have great potential for on-board use. However, they are generally derived in low-fidelity dynamics and hence often struggle to perform in higher-fidelity dynamics. In addition, they are sub-optimal and have user-defined parameters which significantly effect performance [8, 9]. We aim to recover the optimality of these control laws using reinforcement learning (RL) methods. The goal is thus to develop nearly optimal guidance laws for many-revolution low-thrust transfers. We limit the problem to Keplerian dynamics and then verify the performance with eclipses and $J_{2}$ effects as a proof of concept, leaving the task of handling orbit perturbations to future work.

Given the importance stability plays in spacecraft trajectory design, many CLFD control laws are based on Lyapunov control theory [10]. There are many different control laws that use a Lyapunov function to derive the optimal control direction. Joseph [11], Naasz [12] and the Q-law [5, 13] are all such examples. A critical evaluation of these can be found in Hatten's comparison of CLFD control laws [14]. This concluded that Q-law and Ruggiero's DAG [15, 16], a thrust-blending approach, were the most versatile and optimal in terms of time-of-flight for low-thrust transfers.

Several works have further extended the capabilities of these CLFD control laws. In 2008, Maddock and Vasile [17] extended a Lyapunov-based control law to handle both solar radiation pressure and $3^{\text {rd }}$-body effects, however they had trouble with over-shooting and control law chatter. Baresi et al. 2018 [18] derived a Lyapunov controller using relative orbital elements for orbit maintenance around the Martian moon Phobos. In order to account for the gravitational perturbations, they derive the optimal control in a similar fashion to [10]. In 2019, Epenoy and Pérez-Palau [19] designed a Lyapunov-based control law combined with an invariant manifold approach for Earth-Moon transfers. This also included solar radiation pressure and $3^{\text {rd }}$-body effects, however the control direction computed assumes the non-perturbed dynamics control terms will dominate and hence still provide a stable control. They conclude they could improve the Lyapunov-controller by varying the weights.

One method of achieving this is using evolutionary algorithms (EAs). The multi-objective nature of spacecraft trajectory design lends itself to EAs and they still provide many of the benchmarks in the area [20]. Both Lee et al. 2005 [8] and Varga et al. 2016 [9] used a multi-objective genetic algorithm to optimise the Q-law design parameters for a variety of Earth orbit transfers, with the design parameters remaining fixed throughout the transfer. Yang et al. [21] used an artificial neural network and improved cooperative evolutionary algorithm optimiser to make the design parameters of a Q-law state-dependent. The work presented here shares some similarity with this approach, in that they both look to construct state-dependent weights. However, we explore the problem in a different way, utilising the framework of RL as opposed to EAs, with the idea that this will enable us to later embed the effects of perturbations in the control, an aspect that was not considered by them. So in this paper, where we limit to Keplerian with eclipse and $J_{2}$ transfers, we use their results as a comparison for our study.

Reinforcement learning is a promising alternative increasingly used in astrodynamics [20]. Gaudet, Furfaro and Linares have demonstrated the capability of RL for use in Mars landers [22] and asteroid hovering missions [23]. Miller and Linares [24] provided insights into using RL in the low-thrust circular restricted three-body problem, whilst Furfaro and Scorsoglio [25-27] have used it to make the parameters of a Zero-Effort-Miss/Zero-Effort-Velocity (ZEM/ZEV) guidance algorithm state-dependent. In the field of space mission design, Harris et al. 2019 [28] use the RL framework to address high-level mission planning and decision making problems. Perhaps one of the reasons is RL has similarities with both optimal control theory and certain global optimisers such as ant colony optimisation [29], which are widely and successfully used in astrodynamics.

In this work we incorporate state-dependent parameters in the Lyapunov-based Q-law using an advantage actor-critic (AC) reinforcement learning implementation to extend the versatility and improve the optimality of the results. This approach is inspired by a similar approach developed for landing guidance and relative motion problems using the ZEM/ZEV guidance algorithm [26, 27, 30]. We focus on transfers from geostationary transfer orbit (GTO) to geosynchronous orbit (GEO). These are of particular relevance for full-electric GEO platforms and are challenging owing to the large changes in orbital elements, particularly in eccentricity and inclination, and because of the comparisons available in the literature. The transfers are discretised into time-intervals where the parameters can be determined by the learning process and then remain constant over the time-interval. This results in a functional variation along the transfer which is learnt using a single-layer AC approach.

The paper is structured as follows. In Section $\Pi$ an overview of the necessary background is given for both the Q-law and RL. After a detailed description of the implementation and actor-critic algorithm in Section III. we consider time-optimal and mass-optimal GTO-GEO transfer trajectories in Section IV] The conclusions are given in Section $\nabla$ followed by the appendices, acknowledgements and references. 


\section{Background}

In this section we give a brief overview of the two key building blocks for the proposed method: the Q-law in Section II.A and reinforcement learning in Section II.B.

\section{A. Q-law Overview}

One of the most versatile CLFD control laws is the Q-law developed by Petropoulos [5, 13]. Here we discuss the formulation in classical orbital elements (COEs), although we note an alternate formulation in modified equinoctial elements does exist [9]. It is based on a candidate Lyapunov function and is best thought of as a weighted, squared summation of the time required to change the current state to the target state. It is defined as:

$$
Q=\left(1+W_{P} P\right) \sum_{\mathcal{X}} W_{\mathcal{X}} S_{\mathcal{X}}\left(\frac{\delta \mathcal{X}}{\max _{v}(\dot{X})}\right)^{2}, \quad X=a, e, i, \Omega, \omega .
$$

Here, $\mathcal{X}$ represents the set of COEs: semi-major axis $a$, eccentricity $e$, inclination $i$, right ascension of the ascending node (RAAN) $\Omega$ and argument of periapsis $\omega$. The fast variable such as the true anomaly, $v$, is not used in the Q-law formulation. In addition, $W_{P}$ and $W_{\mathcal{X}}$ are weighting factors, $P$ is a penalty function, $S_{\mathcal{X}}$ scaling functions, and $\delta \mathcal{X}=\mathcal{X}-\mathcal{X}_{\mathrm{T}}$ for $\mathcal{X}=a, e, i$ and $\delta \mathcal{X}=\arccos \left(\cos \left(\mathcal{X}-\mathcal{X}_{\mathrm{T}}\right)\right)$ for $\mathcal{X}=\Omega, \omega$. The weights $W_{\mathcal{X}}$ can be used to prioritise targeting particular elements $\mathcal{X}$, changing the transfer characteristics, but need to be positive to guarantee stability.

The derived control minimises the rate of change of the Lyapunov function, hence resulting in a trajectory for the given Lyapunov function formulation. Individual orbital element changes can be targeted, as well as a specific subset of elements. Lyapunov's second theorem states that for a system $\dot{\boldsymbol{Z}}=\boldsymbol{f}(\boldsymbol{Z}), \boldsymbol{Z}=\boldsymbol{X}-\boldsymbol{X}_{\mathrm{T}}$, the equilibrium point $\boldsymbol{X}_{\mathrm{T}}$ is globally asymptotically stable if there exists a scalar Lyapunov function $V(\boldsymbol{Z})$ such that $V(\mathbf{0})=0$; it is positive-definite $(V(\boldsymbol{Z})>0, \forall Z \neq \mathbf{0}) ; \dot{V}(\boldsymbol{Z})<0$; and $\lim _{|\boldsymbol{Z}| \rightarrow \infty}, V(\boldsymbol{Z})=\infty[10]$. For a trajectory optimisation problem, the optimal trajectory can be found by minimising $\dot{V}$. Note we can write the dynamics of the system in matrix form as $\dot{\boldsymbol{X}}=\boldsymbol{B} \boldsymbol{u}$, where $\boldsymbol{u}$ represents the control vector and acts as a perturbing acceleration to the system, and $\boldsymbol{B}$ is a $(5 \times 3)$ matrix representing Gauss's Variational Equations (GVEs) [31]. For an autonomous system,

$$
\dot{V}(\boldsymbol{Z})=\dot{V}\left(\boldsymbol{X}-\boldsymbol{X}_{\mathrm{T}}\right)=\frac{\partial V}{\partial \mathcal{X}} \dot{\boldsymbol{X}}=\frac{\partial V}{\partial \mathcal{X}} \boldsymbol{B} \boldsymbol{u}
$$

In order to drive the system in state $\mathcal{X}$ towards the equilibrium state $\mathcal{X}_{\mathrm{T}}$, we minimise $\dot{V}$ - in the Q-law notation $\dot{Q}$ - by setting the control such that:

$$
\boldsymbol{u}=-\boldsymbol{B}^{T}\left(\frac{\partial Q}{\partial \mathcal{X}}\right)^{T} .
$$

Equation (3) is used to determine the optimal thrust direction for the spacecraft $\hat{\boldsymbol{u}}=\boldsymbol{u} /\|\boldsymbol{u}\|$, whilst the magnitude is given by $f=T / m$, using an engine thrust $T$ and spacecraft mass $m$. The spacecraft's control direction can be described by two angles, the in-plane angle $\alpha$ and the out-of-plane angle $\beta$. The resulting thrust direction in the spacecraft's radial, transverse and out-of-plane (RTN) frame is given by:

$$
\boldsymbol{u}=\frac{T}{m}\left(\begin{array}{c}
\sin (\alpha) \cos (\beta) \\
\cos (\alpha) \cos (\beta) \\
\sin (\beta)
\end{array}\right) .
$$

The expressions $\max _{v}(\dot{X})$ are the maximum rate of change of each COE over the current osculating orbit and can be calculated analytically for all elements except $\omega$. We briefly discuss them here but the reader is encouraged to look at [5, 13, 14] for more information. Starting from the GVEs, the optimal thrust angles for a given location along the orbit, $\nu$, are found by setting $\partial \dot{X} / \partial \alpha=0$ and $\partial \dot{X} / \partial \beta=0$. The optimal location along the orbit for each element is then found by setting $\partial \dot{X} / \partial v=0$ and solving for $v$. The maximum rates of change for $a, e, i$ and $\Omega$ are given by:

$$
\begin{gathered}
\dot{a}=2 f \sqrt{\frac{a^{3}(1+e)}{\mu(1-e)},} \\
\dot{e}=\frac{2 p f}{h},
\end{gathered}
$$




$$
\begin{gathered}
\dot{i}=\frac{p f}{h\left(\sqrt{1-e^{2} \sin ^{2}(\omega)}-e|\cos (\omega)|\right)}, \\
\dot{\Omega}=\frac{p f}{h \sin (i)\left(\sqrt{1-e^{2} \cos ^{2}(\omega)}-e|\sin (\omega)|\right)} .
\end{gathered}
$$

Here $p=a\left(1-e^{2}\right)$ and $h=\sqrt{\mu p}$. The expression is more complex for $\omega$ because it can be changed by both in-plane and out-of-plane components. Petropoulos [13] handled this by treating the two components separately and combining the weighted average of in- and out-of-plane rates: $\max _{v}(\dot{\omega})=\left(\max _{v}\left(\dot{\omega}_{\text {in-plane }}\right)+b \max _{v}\left(\dot{\omega}_{\text {out-of-plane }}\right)\right) /(1+b)$, using:

$$
\begin{gathered}
\dot{\omega}_{\text {in-plane }}=\frac{f}{e h} \sqrt{p^{2} \cos ^{2}(v)+(p+r)^{2}\left(1-\cos ^{2}(v)\right)}, \\
\dot{\omega}_{\text {out-of-plane }}=\frac{p f|\cos (i)|}{h \sin (i)\left(\sqrt{1-e^{2} \cos ^{2}(\omega)}-e|\sin (\omega)|\right)} .
\end{gathered}
$$

The penalty function $P$ and scaling function $S_{X}$ are:

$$
P=\exp \left[k\left(1-\frac{r_{p}}{r_{p \min }}\right)\right], \quad S_{\mathcal{X}}= \begin{cases}{\left[1+\left(\frac{X-X_{\mathrm{T}}}{m X_{\mathrm{T}}}\right)^{n}\right]^{1 / r}} & X=a \\ 1 & \mathcal{X}=e, i, \Omega, \omega,\end{cases}
$$

where $r_{p}$ denotes the periapsis distance and the other user-defined parameters are nominally set to values of $k=100$, $m=3, n=4, r=2$ and $b=0.01$. The penalty function enforces a minimum-periapsis-radius constraint throughout the transfer, $r_{p \min }=6578 \mathrm{~km}$. The scaling function is used to help prevent non-convergence to the target orbit. Note for a "regular" transfer $P, S_{\mathcal{X}} \approx 1$ and in this work the penalty weight $W_{P}=1$.

Coasting can be introduced to conserve fuel at points where using the engine thrust is inefficient. These times are determined using effectivity parameters, which attempt to quantify the effectivity of changing an orbital parameter at a given point in an orbit compared to the optimum point for changing the same orbital parameter. The absolute and relative effectivity parameters are defined as:

$$
\eta_{a}=\frac{\min _{\alpha, \beta}(\dot{Q})}{\min _{v}\left(\min _{\alpha, \beta}(\dot{Q})\right)}, \quad \eta_{r}=\frac{\min _{\alpha, \beta}(\dot{Q})-\max _{\nu}\left(\min _{\alpha, \beta}(\dot{Q})\right)}{\min _{v}\left(\min _{\alpha, \beta}(\dot{Q})\right)-\max _{v}\left(\min _{\alpha, \beta}(\dot{Q})\right)} .
$$

Threshold values can be set and engine thrusting will only occur when $\eta_{a}>\eta_{a}{ }^{\text {thresh }}$ and $\eta_{r}>\eta_{r}{ }^{\text {thresh }}$. By design this will occur at least instantaneously once per orbit. Studies [8] have also shown that, when varying other Q-law parameters in addition to the effectivity, there is little difference in performance between relative and absolute effectivity, and instead the specific transfer will determine which is more applicable. Hence, in this work the absolute effectivity $\eta_{a}$ is used. Following on from Eq. (2) we can calculate $\min _{\alpha, \beta}(\dot{Q})$ as

$$
\min _{\alpha, \beta}(\dot{Q})=\left(\frac{\partial Q}{\partial \mathcal{X}}\right) \boldsymbol{B} \hat{\boldsymbol{u}}=-\left\|\boldsymbol{B}^{T}\left(\frac{\partial Q}{\partial \mathcal{X}}\right)^{T}\right\|,
$$

where $\hat{\boldsymbol{u}}$ is the thrust direction determined in Eq. (3). For the $\min _{v}\left(\min _{\alpha, \beta}(\dot{Q})\right)$ and $\max _{v}\left(\min _{\alpha, \beta}(\dot{Q})\right)$ terms we have to scan through the possible true anomaly, $v$, values to find the maximum and minimum $\dot{Q}$ for the particular osculating orbit - which is done numerically.

All this increases the Q-law's versatility, and distinguishes it from many alternative techniques [14, 16]. A notable characteristic is the ability of the controller to change one element almost sacrificially in order to make it easier to improve/converge one of the other orbital elements. This truly sets the Q-law apart from many other approaches.

However, there are many design parameters that would originally be left to the user's discretion, which can significantly affect the performance and optimality of the method. In particular, the weights $W_{\mathcal{X}}$ determine the contribution of each targeted orbital element $\mathcal{X}$ to the control and the effectivity threshold $\eta^{\text {thresh }}$ will determine when the spacecraft can thrust. Hence, these can be varied throughout to change the characteristics of the trajectory. These two sets of parameters are what we focus on making state-dependent. 


\section{B. Reinforcement Learning Overview}

The major draw for reinforcement learning algorithms is their performance in unfamiliar environments. The performance of CLFD control laws can thus be increased in an environment where the dynamics are unknown and constantly evolving. This can also help provide robustness to any uncertainties in the environment. For a spacecraft, this could arise from orbit determination uncertainties or thruster misalignment. There is significant interest in the applications of RL for mission design, operations, guidance and control to navigation and even the prediction of the dynamics [20]. However, little work has combined RL with existing control laws for trajectory design, which would result in powerful state-dependent learning process on top of an intelligent controller. There has been success for similar approaches in rendezvous and landing problems [26, 27, 30].

Critical to the understanding of RL are the agent, the policy and the reward. Given the state of the environment, the policy will determine what action the agent takes. In this way the agent explores the environment and rewards provide feedback for a given action during the training process [32]. Hence, the formulation and structure of the reward (or cost) function is critical. During the training process, an agent will take actions based on an untrained policy, and over time this policy will improve until the optimal policy is learnt. This optimal policy maps states to actions to maximise the total reward (or minimise the total cost) for the environment.

More formally, RL problems are usually posed within a Markov decision process (MDP), as sequence of states $x_{i}$, actions $u_{i}$ and a transition dynamics distribution with conditional density $p\left(x_{i+1} \mid x_{i}, u_{i}\right)$, which represents the dynamic relationship between states, given action $u$ and a cost function. The agent (in our case the spacecraft) interacts with the environment using a parametrised policy $\pi_{\vartheta}(u \mid x)$ that defines the action taken $u \sim \pi_{\vartheta}(u \mid x)$. As it interacts with the MDP it collects observations $x_{i}$ and costs $c\left(x_{i}, u_{i}\right)$ based on the actions taken. For the sequence of states, the discounted cost-to-go $C_{i}$ is often used, which is a discounted sum of the cost $c\left(x_{i}, u_{i}\right)$ at each remaining state along the sequence with discount factor $\gamma \in(0,1]$, and is written as:

$$
C_{i}=\sum_{j=i}^{\text {end }} \gamma^{j-i} c\left(x_{j}, u_{j}\right)
$$

The goal of the agent to minimise the performance objective $J\left(\pi_{\vartheta}\right)=\mathbb{E}\left[C_{0} \mid \pi\right]$ by finding the optimal policy $\pi_{\vartheta}^{\star}$. The optimal policy arises from Bellman's equations [32] and is the policy that optimises the value function. There are different ways of defining the value function. The state value function $V^{\pi}(x)$ is defined as:

$$
V^{\pi}(x)=\mathbb{E}\left[\sum_{j=0}^{\text {end }} \gamma^{j} c_{j+1} \mid x_{0}=x, \pi\right]
$$

which depends only on the state and assumes the policy $\pi$ is followed starting from this state. The state-action value function $Q^{\pi}(x, u)$ instead is defined as:

$$
Q^{\pi}(x, u)=\mathbb{E}\left[\sum_{j=0}^{\text {end }} \gamma^{j} c_{j+1} \mid x_{0}=x, u_{0}=u, \pi\right]
$$

and depends both on state and action. However, instead of assuming the action $u$ is generated by the policy $\pi$, the action chosen is a free variable [33]. Once the transition to the next state has been made, the policy $\pi$ determines the subsequent actions $u$. The two value functions can be used to define a quantity called the advantage function:

$$
A^{\pi}(u, x)=Q^{\pi}(x, u)-V^{\pi}(x)
$$

which uses the state value function as a state-dependent baseline to estimate how much better an action is with respect to the expected optimal action under the current policy and is used in general to increase the learning performance. During training, the agent will estimate the cost-to-go function for a given policy in what is called policy evaluation.

For a continuous state and action space, the most popular RL algorithms are policy gradient algorithms [34, 35]. These use the value function to adjust parameters $\vartheta$ of a policy $\pi_{\vartheta}$ in the opposite direction of the gradient of the objective function:

$$
\nabla_{\vartheta} J\left(\pi_{\vartheta}\right)=\mathbb{E}\left[\nabla_{\vartheta} \log \pi_{\vartheta}(u \mid x) Q^{\pi}(x, u)\right] .
$$

So the update of the parameters of the policy is given by:

$$
\vartheta_{k+1}=\vartheta_{k}-\alpha_{k} \nabla_{\vartheta} J_{k}\left(\pi_{\vartheta}\right)
$$


where $\alpha_{k}$ is the learning rate at iteration $k$, and controls the magnitude of the update. The challenge lies in computing the performance objective. In developing the REINFORCE algorithm, Williams [36] showed it was possible to estimate this using samples from experience rather than actually computing it. This resulted in the policy gradient theorem [34], significantly reducing the necessary computation.

Actor-critic algorithms are based on policy gradient methods described above, where there are two main components. A critic evaluates the quality of a given action by approximating the value function using a neural network. The actor learns the policy and hence maps the state to the action the agent should take. For more information, Grondman et al. 2012 [33] provide a good survey of AC approaches. Normally AC algorithms use conventional neural networks to represent both the policy and value functions, and use back-propagation to iteratively learn their weights. This is a multiple step process, and is the brain behind the learning process. Extreme Learning Machines (ELMs) [37] are instead single-layer feed-forward networks (SLFNs) that remove the need for back-propagation, significantly increasing the learning process speed. They assume any continuous target function $f(x)$ can be approximated by a SLFN with a set of $L$ random hidden nodes, activation functions $h(x)$ and learnt parameters $\beta$ as $f_{L}(x)=\sum_{j=1}^{L} \beta_{j} h_{j}(x)$ [37]. It has been shown that it is both feasible and efficient to use an ELM critic in an AC algorithm, and the training time for ELM has little impact on the total training time [26]. Hence we use them in our implementation described below.

\section{Implementation}

In this work, the problem is mostly handled in Keplerian dynamics. In Section III.A we discuss details of implementing the Q-law, and in Section III.B we discuss the specific RL algorithm implemented in this work.

\section{A. Q-law}

The implementation of the Q-law is widely based on Hatten's work [14], and is done in MatLab for prototyping reasons, following the outline given in Section III.A The dynamics are primarily governed by the GVEs and a variable-step Adams-Bashforth-Moulton PECE solver (using MatLab's ode113 integrator) is used to propagate these equations in time. The equation of motion for the mass is generally decoupled from evolution of the COEs. Given an engine with thrust $T$ and specific impulse $I_{s p}$, the rate of change of mass is

$$
\frac{d m}{d t}=-\delta_{e} \frac{T}{g_{0} I_{s p}}
$$

where $g_{0}$ is the reference gravitational acceleration, and $\delta_{e}=1$ if the engine is on, $\delta_{e}=0$ if the engine is off, and $0<\delta_{e}<1$ if the spacecraft is in the eclipse penumbra or briefly when switching between on and off states.

Implementing the thrust effectivity $\eta_{\mathrm{a}}$ can be done using Eq. (8). However, if the instantaneous thrust effectivity $\eta_{\mathrm{a}}$ is below the threshold $\eta_{\mathrm{a}}^{\text {thresh }}$, then the thrust is set to zero and the spacecraft will coast until $\eta_{\mathrm{a}}$ goes back above the threshold. By design, this will occur at least once per orbit. In order to prevent a step function in the ODE integration, a sigmoid function Fis used to transition between states where the thrust is off to those where the thrust is on. We found that $\operatorname{sigmoid}\left(\left(\eta_{\mathrm{a}}-\eta_{\mathrm{a}}^{\text {thresh }}\right) / 5000\right)$ worked well and removed any integration issues.

The control is given by Eq. (3) in the constant weights case. However, when the weights $W_{\mathcal{X}}$ vary, we make the assumption that $\left|\frac{\partial Q_{P}}{\partial W_{X}} \dot{W}_{X}\right|<<\left|\frac{\partial Q_{P}}{\partial X} \dot{X}\right|$ and hence we assume this control still provides a sub-optimal control direction (similar to [19]) and and $\dot{Q}$ can still be expressed as:

$$
\dot{Q}=\frac{\partial Q}{\partial \mathcal{X}} \dot{\mathcal{X}}+\frac{\partial Q}{\partial W} \dot{W} \approx \frac{\partial Q}{\partial \mathcal{X}} \dot{\boldsymbol{X}}=\frac{\partial Q}{\partial \mathcal{X}} \boldsymbol{B} \boldsymbol{u} .
$$

The transfers are discretised into time-intervals over which the weights remain constant such that this assumption holds. In this work, the state-dependent parameters are determined in 1 days intervals.

\section{B. Actor-Critic ELM}

Here we discuss the actor-critic (AC) implementation. This approach is inspired by a similar approach developed for landing guidance and relative motion problems using the ZEM/ZEV guidance algorithm [26, 27, 30]. This acts as an outer learning layer which is able to learn the optimal state-dependence of the Q-law parameters by simulating

\footnotetext{
$* \operatorname{sigmoid}(z)=1 /(1+\exp (-z))$
} 
trajectories in the environment and receiving feedback based on the performance. Unlike in Section II.B, where $x$ is used to denote a general state, here $\mathcal{X}$ is used instead to signify the state is specifically the COE state vector.

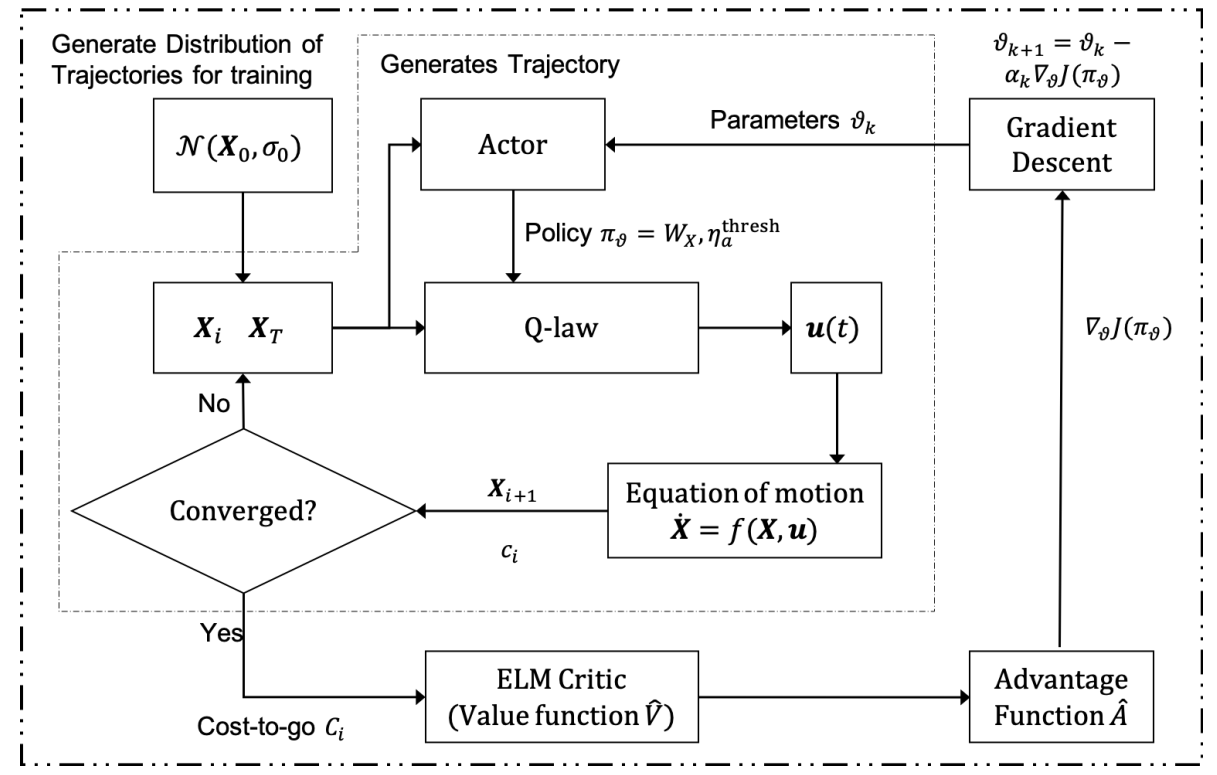

Fig. 1 The proposed actor-critic algorithm for making the Q-law parameters state-dependent

The overall framework is shown in Fig. 1. On its own, the Q-law is a closed-loop control law and given a state $\boldsymbol{X}_{i}$ and target state $\mathcal{X}_{\mathrm{T}}$, can determine the control required as a function of time to drive the state to the target orbit, as seen in the inner loop. What we propose is to discretise the transfer into a set of time-intervals, between which the Q-law parameters can be modified to change the characteristics and optimality of the transfer. This involves an actor network which will take the current state as an input and return the Q-law parameters $W_{\mathcal{X}}$ and $\eta_{a}^{\text {thresh }}$ according to a policy $\pi_{\vartheta}$. In this work, the time-step between changing the parameters is 1 day. The trajectory is said to converge when it is within a convergence criteria of the target orbit, or the maximum integration time-steps have been reached.

In order to learn the parameters of the actor network, and in turn the state-dependent nature of the Q-law parameters, we add an outer learning loop to improve them with each iteration $k$. Using the AC formulation discussed in Section II.B. a batch of $N$ trajectories need to be generated within each iteration. Each trajectory is simulated using the same policy $\pi_{\vartheta}$ until it converges. In this work, the initial states have a Gaussian distribution in position and velocity of root-mean-squared (RMS) $0.3 \mathrm{~km}$ and $3 \mathrm{~m} / \mathrm{s}$ to allow for state-space exploration. At each time-step $i$ during the trajectory we assign a cost $c_{i}$ to each episode based on the performance in the time-interval $i$ to $i+1$. Importantly, there is a stochastic nature to the policy during training to ensure sufficient exploration of the environment: a Gaussian distribution about the actor output with standard deviation $\sigma_{k}$.

Once all the episodes in a batch have converged or terminated, we can calculate a cost-to-go $C_{i}$ at each state and for each episode. The critic network uses these states and cost-to-go pairs to find the approximate value, $\hat{V}^{\pi}$, of each state under the current policy $\pi_{\vartheta}(\boldsymbol{u} \mid \boldsymbol{X})$. This is then used to obtain the approximate advantage function

$$
\hat{A}_{i}^{\pi}=\hat{Q}^{\pi}-\hat{V}^{\pi}=c_{i}+\gamma \hat{V}^{\pi}\left(\mathcal{X}_{i+1}\right)-\hat{V}^{\pi}\left(\mathcal{X}_{i}\right) .
$$

Here $\hat{V}^{\pi}\left(\mathcal{X}_{i}\right)$ indicates the expected cost-to-go of the current state - its value - and $\hat{V}^{\pi}\left(\mathcal{X}_{i+1}\right)$ the expected cost-to-go of the subsequent state. Both are approximate given they are learnt by the critic network. The difference between the two is the expected cost of the current state $\mathcal{X}_{i}$. Hence the advantage function reflects how much better the action $\boldsymbol{u}_{i}$ taken in state $\mathcal{X}_{i}$ is with respect to the expected action, which can be determined by having multiple trajectories within the same batch during the learning process. We note this can be modified to an $n$-steps advantage function [26], in order to reduce the effect of any bias, but this is not done in this work.

Updating the parameters $\vartheta_{k}$ is then done using gradient descent, $\vartheta_{k+1}=\vartheta_{k}-\alpha_{k} \nabla_{\vartheta} J_{k}\left(\pi_{\vartheta}\right)$, where

$$
\nabla_{\vartheta} J\left(\pi_{\vartheta}\right) \approx \frac{1}{N} \sum_{n=1}^{N} \sum_{i=1}^{T} \nabla_{\vartheta} \log \pi_{\vartheta, n, i} \hat{A}_{n, i}
$$


and $T$ represents the total number of time-steps needed (i.e. the end of the trajectory). Here $\nabla_{\vartheta} \log \pi_{\vartheta, n, i}$ is the gradient of the log-probability of the stochastic policy for episode $n$ at time-step $i$. As mentioned above, a stochastic policy ensures sufficient exploration of the state-space during training. For a Gaussian policy with mean $\mu_{i}$ and stochasticity $\sigma_{k}$, this log-probably is analytically given by:

$$
\nabla_{\vartheta} \log \pi_{\vartheta, i}=\frac{\pi_{\vartheta, i}-\mu_{i}}{\sigma_{k}^{2}} \psi\left(\boldsymbol{X}_{i}\right) .
$$

The actor used is a SLFN, which takes the state as input and uses radial basis functions (RBF) to give the Q-law weights as output - see Fig. 2a. These RBFs act as the network's features and their centres, $\boldsymbol{c}_{\mathcal{X}}$, are evenly distributed across the state-space in a similar manner to [25]. Different states trigger different features to various extents depending on how much the state overlaps with the grid of features, as can be seen in the following equation

$$
\psi(\boldsymbol{X})=e^{-\beta_{X}\left\|\boldsymbol{X}-\boldsymbol{c}_{X}\right\|^{2}} .
$$

In this work, we divide the state-space into grids over $\{a, e, i\}$ using 20 intervals within each grid. This ensures approximately 10,000 features cover the state space, however not all of these are activated during the training process. As the features are specifically designed for the problem, only the second set of weights in the actor neural network, $\vartheta$, need to be learnt. This is advantageous as it reduces the complexity and helps speed up the learning process. However, it can result in less nuance in the behaviour. In order to bound the outputs of the actor network, a $1 / 2(\tanh (x)+1)$ function is used, ensuring $0<W_{\mathcal{X}}, \eta_{\mathrm{a}}<1$. Given this setup, the weights of the network, $\vartheta$, are initialised as a Gaussian distribution with mean 0 and variance 0.25 .

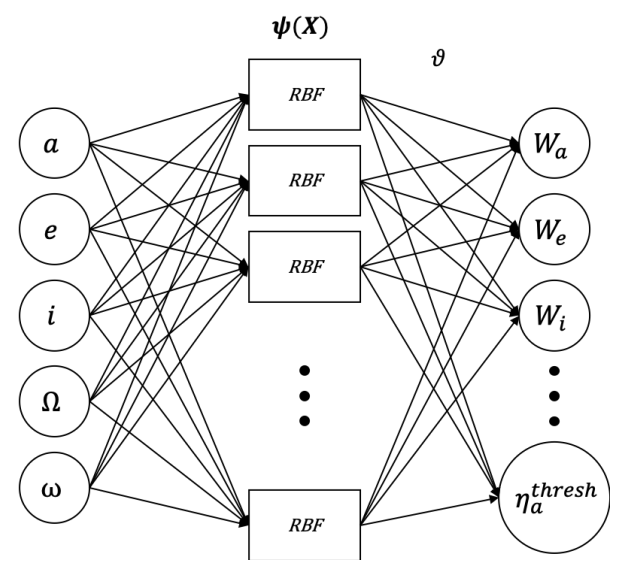

(a) Actor network

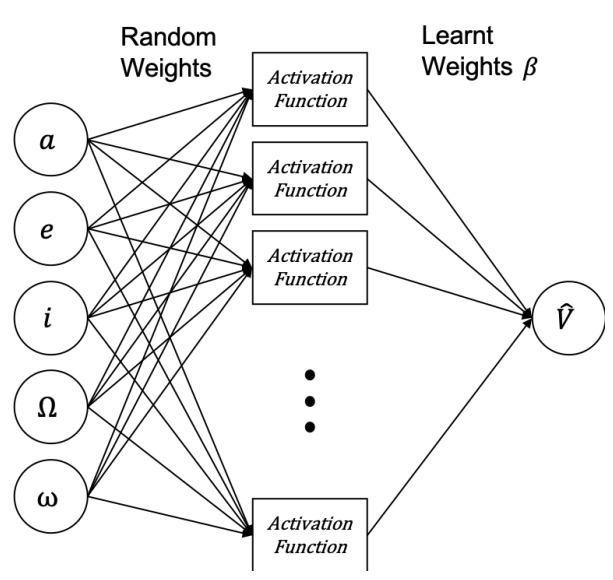

(b) Critic network

Fig. 2 Structures of the actor and critic networks. Both are single-layer feed-forward networks.

The structure of the critic network is shown in Fig. 2b and is the form required for an ELM set-up [37]. The weights in the first layer of neurons are random, whilst the second layer weights, $\beta$, are learnt within each iteration of the algorithm. Unlike traditional critic networks, where the weights are learnt iteratively, this ELM approach means the approximation of the cost-to-go, the value function $\hat{V}$, is known to good knowledge at each iteration. This allows for a faster learning process. In this work, a sigmoid activation function is used, with 4000 hidden features ensuring the function approximation is sufficiently accurate at each iteration. This can be monitored in terms of root-mean-square error. Increasing the number of time-steps per episode helps to decrease the error in the function approximation [26].

In order to ensure learning convergence, a tanh functional dependence of the learning rate $\alpha_{k}$ and stochasticity $\sigma_{k}$ are used. As the cost function reduces, the values of $\alpha_{k}$ and $\sigma_{k}$ decrease to help with the exploration and exploitation dilemma. More advanced update methods such as the ADAM optimiser [38] were considered but for this application the functional dependence appeared to be more suitable. Finally, an update threshold $\max \left|\alpha_{k} \nabla_{\vartheta} J_{k}\left(\pi_{\vartheta}\right)\right|<0.05$ was set to ensure greater stability in the learning process and no one iteration dominates the form of $\vartheta$. 


\section{Results}

In this work, we focus on low-thrust transfers from GTO-GEO. This is done in Keplerian dynamics and later including eclipse and $J_{2}$ effects. However, it's important to note the dynamics embedded in the Q-law remain Keplerian for all cases. This means we can explore the low-fidelity control in a higher-fidelity environment (eclipse and $J_{2}$ effects) it doesn't have knowledge of. We consider both time-optimal and mass-optimal transfers. The results for the AC Q-law implementation are compared to those found in literature, the classical/nominal Q-law case, and simulations run using a particle swarm optimiser (PSO). In the case of the PSO, we run two different simulations referred to as Q-law PSO fixed and Q-law PSO time-dependent in the results summary. In the first case we optimise the Q-law weights and keep them constant over the transfer. For the second case, we discretise the transfer into fixed time-intervals of 5 days and use the PSO to optimise the Q-law weights for each time-interval, parametrising the weights as piece-wise constant over a grid of time-intervals.

For the AC Q-law approach, defining the cost function for a particular objective is key. In the time-optimal case, the cost function is a $\delta$-function of the final time-of-flight (ToF) for the transfer, along with a penalty term which ensures the trajectory converges within the allowed integration time. Using $\mathcal{X}_{T}$ as the targeted orbital elements and $\mathcal{X}_{\text {conv }}$ to denote the convergence criteria for each element, we can write the cost function as:

$$
c_{i}^{\mathrm{ToF}}=\delta\left(\boldsymbol{X}_{i}-\mathcal{X}_{f}\right)\left(\mathrm{ToF}+\sum_{\mathcal{X}=a, e, i}\left(\frac{\mathcal{X}_{f}-\mathcal{X}_{T}}{\mathcal{X}_{\mathrm{conv}}}\right)^{2}\right) .
$$

In the mass-optimal case, the cost function is more complex, and has two terms which are state-dependent. The first is the propellant used over the decision step, $\left(m_{i+1}-m_{i}\right)$. The second is an estimate of total time-of-flight. We used this to inform the system if the current transfer is expected to overshoot the desired time-of-flight, ToF $F^{\text {aim }}$. Fixing the time-of-flight is needed for literature comparisons. Using the ratio of $\delta \mathcal{X}$ to $\max _{v}(\dot{\mathcal{X}})$, the Q-law can act as an estimate for the remaining time-to-go for the transfer squared. This is only an estimate as $\max _{v}(\dot{\mathcal{X}})$ is orbit dependent and hence can increase or decrease throughout the transfer, and of course the GVEs are nonlinear. Here we denote the estimate of the total time-of-flight at state $\mathcal{X}_{i}$ as $\mathrm{ToF}_{i}^{\text {est }}$ :

$$
\mathrm{ToF}_{i}^{\mathrm{est}}=t_{i}+\sqrt{\sum_{X=a, e, i}\left(\frac{\delta X_{i}}{\max _{v}\left(\dot{X}_{i}\right)}\right)^{2}} .
$$

Both time-of-flight terms are only included if the transfer time-of-flight is greater than the targeted time-of-flight, $\mathrm{ToF}^{\mathrm{aim}}$. Again there is a penalty term which ensures the trajectory converges within the allowed integration time. Hence, we can write the cost function as:

$$
\begin{gathered}
c_{i}^{\text {mass }}=\left(1-\delta\left(\mathcal{X}_{i}-\mathcal{X}_{f}\right)\right)\left(\left(m_{i+1}-m_{i}\right)+\frac{\left(\mathrm{ToF}_{i+1}^{\mathrm{est}}-\mathrm{ToF}^{\mathrm{aim}}\right)^{2}}{\mathrm{ToF}^{\mathrm{aim}}}\left(\mathrm{ToF}_{i+1}^{\mathrm{est}}>\mathrm{ToF}^{\mathrm{aim}}\right)\right)+ \\
\delta\left(\boldsymbol{X}_{i}-\boldsymbol{X}_{f}\right)\left(\left(\mathrm{ToF}-\mathrm{ToF}^{\mathrm{aim}}\right)^{2}+\sum_{\mathcal{X}=a, e, i}\left(\frac{\mathcal{X}_{f}-\mathcal{X}_{T}}{\mathcal{X}_{\text {conv }}}\right)^{2}\right)
\end{gathered}
$$

For both the time- and mass-optimal transfers studied, the best performance was found using a discount factor of $\gamma=1$ (i.e. no discount in the cost-to-go). Given the reliance on a $\delta$-function at the final state in both cost functions, using $\gamma=1$ ensures this has an effect on each state in the transfers not just the end states. However, when the cost function has a more state-dependent nature, a discount factor of $\gamma \sim 0.99$ would be more appropriate. We hope to investigate this in future work.

\section{A. GTO-GEO transfer in Keplerian Dynamics - Case A}

Here we consider a GTO-GEO transfer with an inclination change in Keplerian dynamics. The parameters are chosen to allow comparison with Yang et al. 2016 [21] and Geffory and Epenoy 1997 [39]: the modelled spacecraft has mass $=2000 \mathrm{~kg}$, thrust $=0.35 \mathrm{~N}$ and Isp $=2000 \mathrm{~s}$. The initial and target orbits are shown in Table 11 along with the convergence criteria. These convergence criteria prevent the Q-law chattering close to the target orbit and are chosen to allow comparison with the literature. The results for the time-optimal and mass-optimal cases are compared to both results found in the literature and benchmarks obtained using a PSO and Q-law - see Table 2. 
Table 1 Initial and target orbital elements, and convergence criteria, for a GTO-GEO transfer

\begin{tabular}{lcccccc}
\hline & $a(\mathrm{~km})$ & $e$ & $i(\mathrm{deg})$ & $\Omega(\mathrm{deg})$ & $\omega(\mathrm{deg})$ & $v(\mathrm{deg})$ \\
\hline \hline Initial & $24,505.9$ & 0.725 & 7 & 0.0 & 0.0 & 0.0 \\
Target & $42,165.0$ & 0 & 0 & free & free & free \\
Convergence & $42(0.1 \%)$ & $8.5 \mathrm{e}-4$ & 0.1 & - & - & - \\
\hline
\end{tabular}

For the time-optimal transfer, a batch size of $N=12$ was used, with a learning rate $\alpha=0.02$ and stochasticity $\sigma=0.03$. The evolution of the orbital elements $a, e, i$ as well as the spacecraft mass $m$ are shown in Fig. 3a The corresponding trajectory is visualised in Fig. 3b. Fig. 3c shows the evolution of the weights, which are the parameters learnt by the AC algorithm and hence made state-dependent. However, they don't tell the full story regarding which components are prioritised. Fig 3e plots the evolution of the Q-law throughout the transfer. As discussed in Section II.A. the Q-law can be thought of as a sum of the squared time-to-go to change each orbital element to its target value. Hence, we can see that the eccentricity is the major contributor to the overall Q-law value. However, the combination of Figs. 3c and $3 \mathrm{~d}$ result in Fig $3 \mathrm{e}$. Hence, we learn that the classical Q-law over emphasises the importance of the eccentricity, and instead, the contribution of both the inclination and semi-major axis need to be increased, particularly early on in the transfer, for a time-optimal solution. The classical Q-law is also known to overshoot the semi-major axis in order to make the inclination change less expensive [7, 14]. However, this sacrifices time-of-flight and hence we see this trait removed when optimising for time-of-flight. Figure $3 \mathrm{f}$ ] shows the average and best time-of-flight within each batch of trajectories during the learning process. The time-of-flight was 138.35 days, within $1.0 \%$ of the result found in [21].

Table 2 Comparison of state-dependent Q-law performance for GTO-GEO transfer, using a spacecraft with mass $2000 \mathrm{~kg}$, engine thrust $0.35 \mathrm{~N}$ and Isp 2000s.

\begin{tabular}{llcc}
\hline Objective & Method & Time of Flight (days) & Propellant $(\mathrm{kg})$ \\
\hline \hline Time-of-flight & Yang et al. 2016 [21] & 137.3 & 211.72 \\
& Q-law nominal $W_{\mathcal{X}}=1$ & 144.18 & 222.29 \\
& Q-law PSO fixed $W_{\mathcal{X}}$ & 138.54 & 213.61 \\
& Q-law PSO time-dependent $W_{\mathcal{X}}$ & 137.67 & 212.11 \\
& Q-law AC state-dependent $W_{\mathcal{X}}$ & 138.35 & 213.32 \\
\hline Propellant & Yang et al. 2016 [21] & 150 & 187.97 \\
& Geffroy \& Epenoy 1997 [39] & 150 & 192 \\
& Q-law PSO fixed $W_{\mathcal{X}}, \eta$ & 150 & 195.46 \\
& Q-law PSO time-dependent $W_{\mathcal{X}}, \eta$ & 150 & 193.16 \\
& Q-law AC state-dependent $W_{\mathcal{X}}, \eta$ & 150 & 191.84 \\
\hline
\end{tabular}

Figure 4 shows a similar breakdown for the mass optimal transfer, where a batch size of $N=24$ was used to increase learning stability. Both the learning rate $\alpha=0.02$ and stochasticity $\sigma=0.03$ were the same as in the time-optimal case. For literature comparison, the time-of-flight was fixed to 150 days, and the propellant used to reach the target orbit is then minimised. In Fig $4 \mathrm{~b}$ the grey regions indicate coast arcs, whilst the red regions indicate full engine thrusting. For this particular GTO-GEO transfer, we are increasing the altitude of periapsis, which is most effectively done in the apoapsis region. Using a fixed-time, mass-optimal approach proved challenging for the learning process, as the constraint of fixing a transfer time and minimising propellant can lead to conflicting improvements. This was solved using the state-dependent estimate of the time-to-go seen in the cost function - see Eq. 24). Figure 4c includes the evolution of $W_{\mathrm{sma}}, W_{\mathrm{e}}$ and $W_{\mathrm{inc}}$ as for the mass-optimal case, as well as the evolution of the effectivity parameter $\eta_{\mathrm{a}}^{\text {thresh }}$. This allows the algorithm to switch between thrust and coast arcs during the transfer. For the mass-optimal transfer we find a solution within $2.1 \%$ of [21] but on-par with the orbit averaging approach in [39]. 

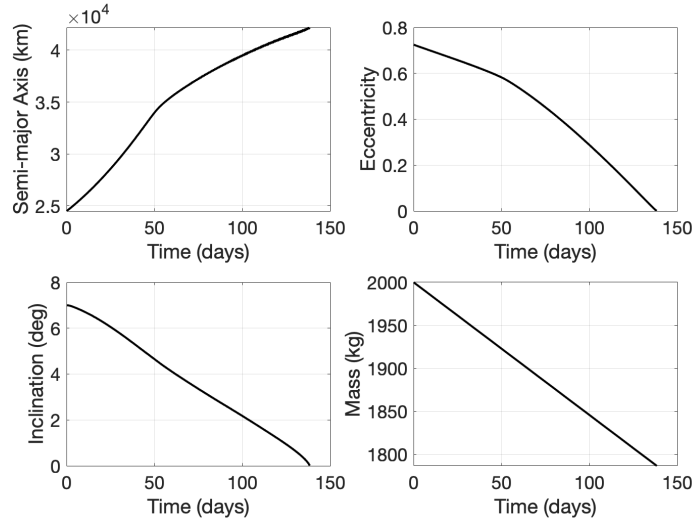

(a) Orbital Parameters

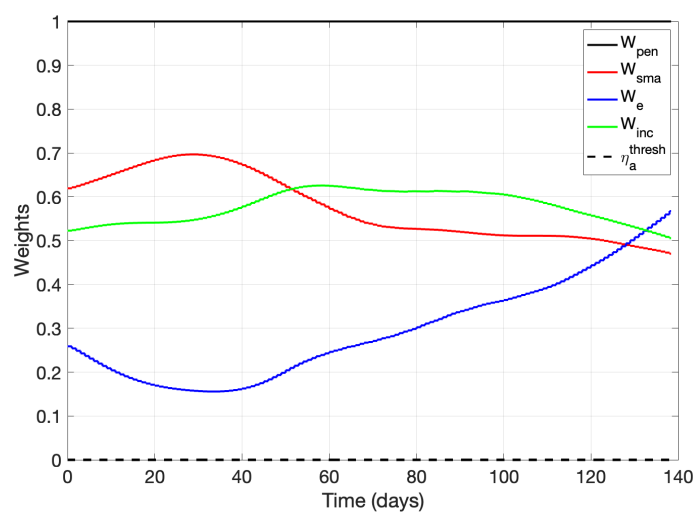

(c) Weights

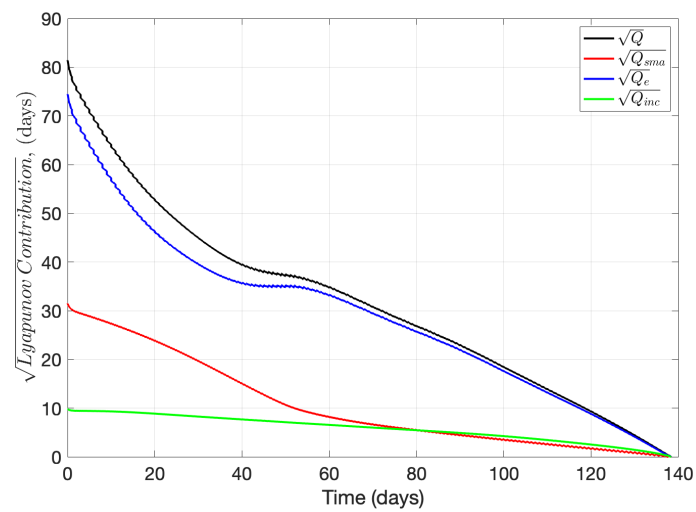

(e) Q-law Evolution

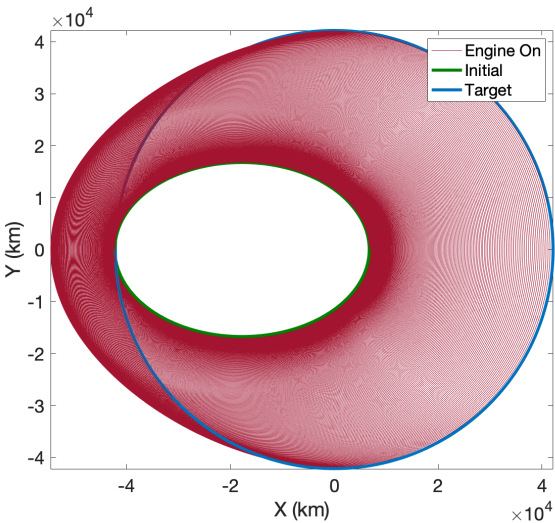

(b) Trajectory

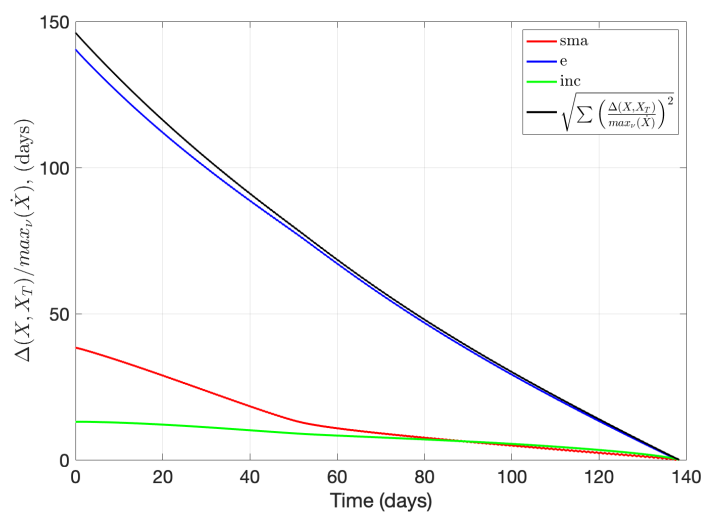

(d) Estimate of the time-to-go from the orbital elements

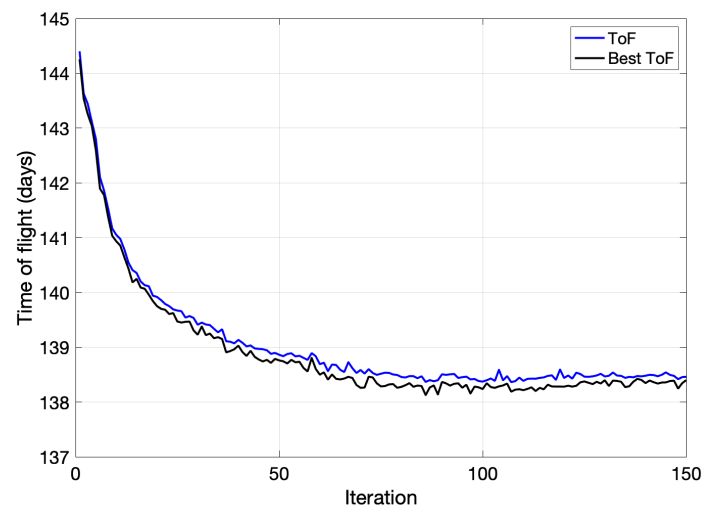

(f) Learning Process

Fig. 3 Time-optimal GTO-GEO transfer using a Q-law with state-dependent weights in Kelperian dynamics. 

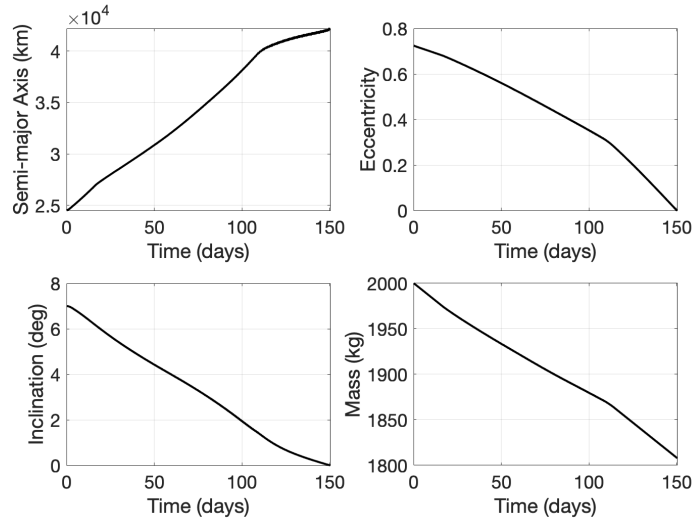

(a) Orbital Parameters

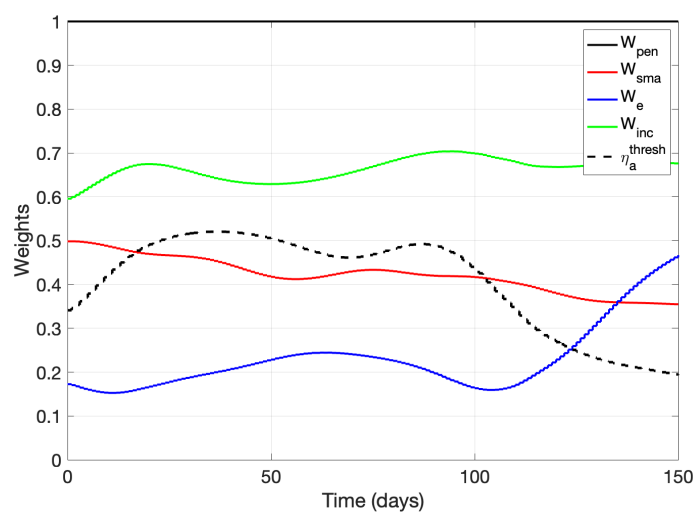

(c) Weights

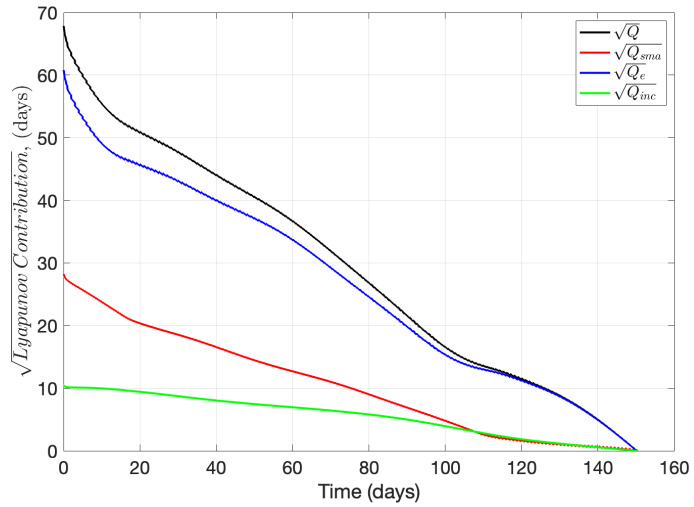

(e) Q-law Evolution

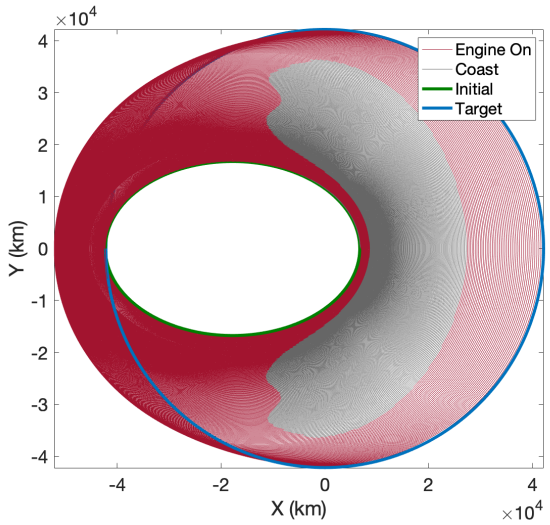

(b) Trajectory

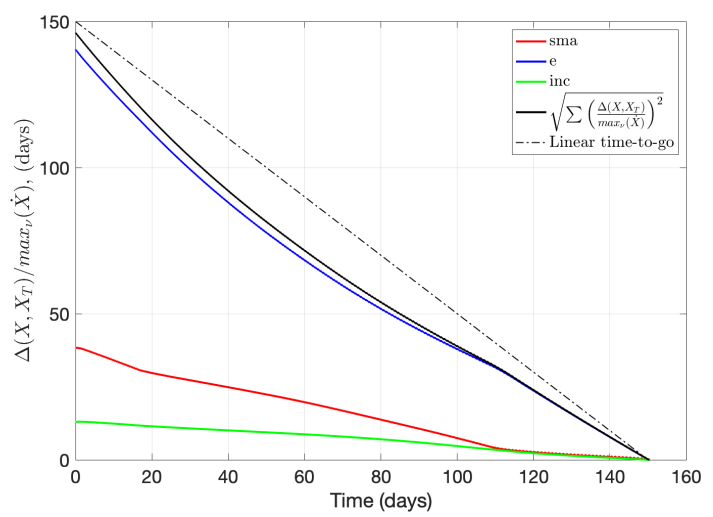

(d) Estimate of the time-to-go from the orbital elements

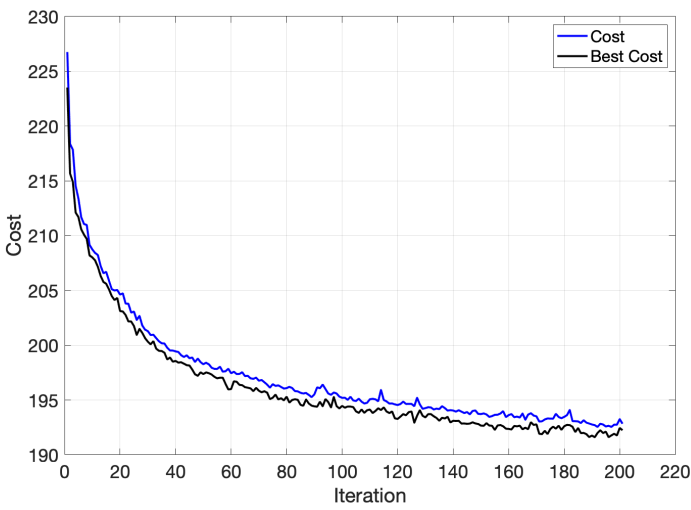

(f) Learning Process

Fig. 4 Mass-optimal GTO-GEO transfer using a Q-law with state-dependent weights and thrust effectivity threshold in Kelperian dynamics. 


\section{B. GTO-GEO transfer in Keplerian Dynamics - Case B}

In the previous section the chosen test case allows comparisons with an orbit averaging approach in [39] and a state-dependent parameter approach using EAs in [21]. Here we consider a separate GTO-GEO transfer to allow for a direct comparison with a time-optimal transfer computed by solving the optimal control problem with multiple shooting methods. The comparison comes from Topputo and Ceccherini's work in [40]. There they solve a GTO-GEO transfers with a plane change using low-thrust parameters. Here we attempt to replicate their solution using our technique, in order to indicate how well this state-dependent AC approach compares with the known optimal solution.

Unfortunately a complete comparison is difficult as they don't define any convergence criteria or tolerance within which the target orbit can be reached. This process is very common when using targeting algorithms, particularly the Q-law, as they are prone to chattering when in proximity of the target orbit. This chattering is avoided by setting a convergence criteria which terminates the transfer before control law chatter occurs. In reality, this is sufficient for mission design because alternative control mechanisms, particularly those involving rendezvous capabilities, can take over in the closing stages of a transfer, and drive the spacecraft towards the target orbit. However, here it artificially reduces the transfer length and hence the comparison is not ideal. Table 3 shows the initial and target orbit, along with the selected convergence criteria for the learning and validation process. During the learning process, the convergence criteria is more relaxed in order to aid state-space exploration without causing too much control law chatter. Subsequently the results are further validated using a stricter convergence criteria, one which the nominal Q-law is unable to converge too. Not only does this allow for better comparison with the optimal control solution, but it also shows that state-dependent weights reduce the likelihood of control law chatter.

Table 3 Initial and target orbital elements, and convergence criteria, for a GTO-GEO transfer

\begin{tabular}{lcccccc}
\hline & $a(\mathrm{~km})$ & $e$ & $i(\mathrm{deg})$ & $\Omega(\mathrm{deg})$ & $\omega(\mathrm{deg})$ & $v(\mathrm{deg})$ \\
\hline \hline Initial & $24,417.5$ & 0.726794 & 5 & 0.0 & 0.0 & 0.0 \\
Target & 42,164 & 0 & 0 & free & free & free \\
Convergence (Learning) & 10 & 0.005 & 0.05 & - & - & - \\
Convergence (Validation) & 2 & 0.002 & 0.02 & - & - & - \\
\hline
\end{tabular}

Table 4 Comparison of state-dependent Q-law performance for GTO-GEO transfer, using a spacecraft with mass $2000 \mathrm{~kg}$, engine thrust $0.2 \mathrm{~N}$ and Isp $2000 \mathrm{~s}$.

\begin{tabular}{lccc}
\hline Method & Time of Flight (days) & Q-law Residual $(\sqrt{Q})($ days $)$ & Convergence Criteria \\
\hline \hline Topputo \& Ceccherini [40] & 241.96 & - & - \\
Q-law nominal $W_{\mathcal{X}}=1$ & 249.19 & 0.26 & Learning \\
Q-law AC state-dependent $W_{\mathcal{X}}$ & 241.08 & 0.55 & Learning \\
Q-law nominal $W_{\mathcal{X}}=1$ & no convergence & 0.07 & Validation \\
Q-law AC state-dependent $W_{\mathcal{X}}$ & 241.58 & 0.23 & Validation \\
\hline
\end{tabular}

Table 4 shows the performance of our approach compared with both the nominal Q-law performance and the optimal control solution. Using a normal convergence criteria, the state-dependent Q-law can improve the nominal Q-law transfer by more than 8 days, a $3 \%$ improvement. The time-of-flight of 241.08 days is actually slightly better than the optimal control solution. This is possible because the convergence criteria artificially reduces the transfer length. As discussed earlier, the Q-law can act as a time-to-go estimator. Hence, the residual Q-law value gives an indication of the approximate remaining transfer time squared. Combining this with the actual time-of-flight gives 241.63 days, within $0.14 \%$ of the known optimal solution. However, this Q-law residual neglects the challenges of converging to the target orbit and is only an estimate. What it does demonstrate, however, is the capability of the proposed approach and how close it is to the optimal control solution.

Using a stricter convergence criteria causes the nominal Q-law to chatter when trying to converge. Using the trained state-dependent weights, the Q-law is able to converge after 241.58 days. Again, with the residual Q-law value, this gives a combined time-of-flight of 241.81 days, within $0.07 \%$ of the known solution. 


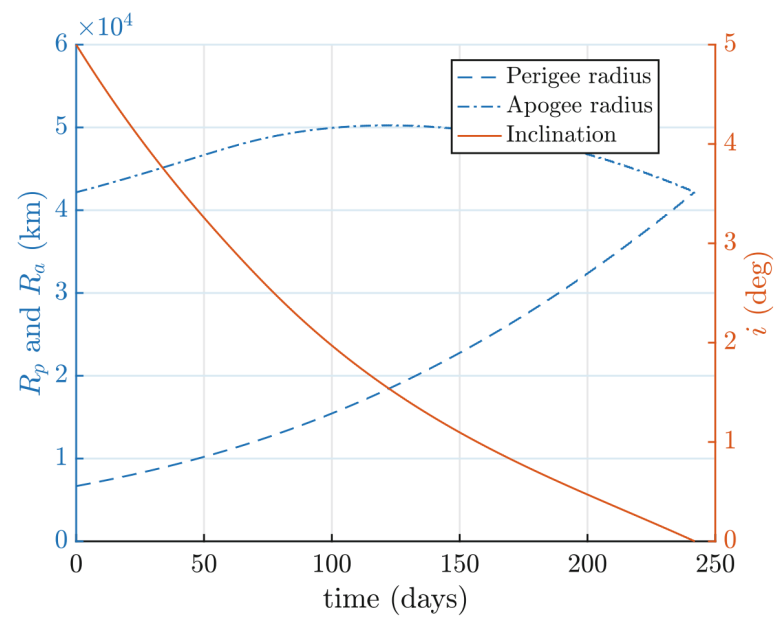

(a) Optimal control solution (taken from [40])

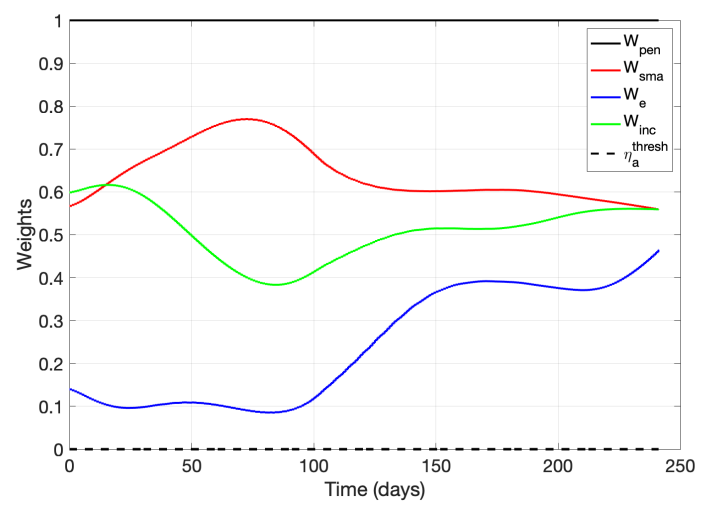

(c) Weights

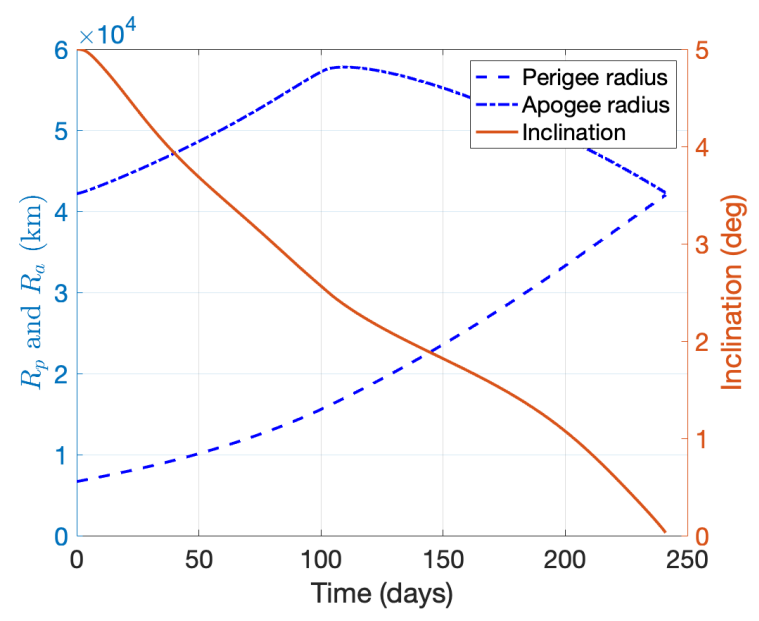

(b) State-dependent Q-law

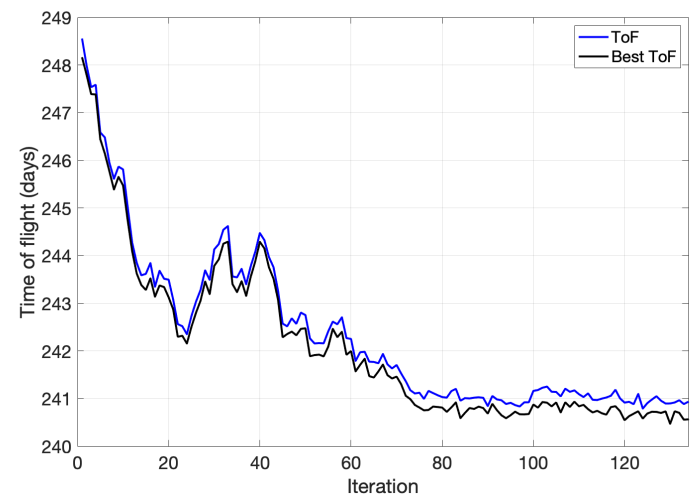

(d) Learning Process

Fig. 5 Time-optimal GTO-GEO transfer using a Q-law with state-dependent weights in Kelperian dynamics and comparison with optimal control solution.

Figures $5 \mathrm{a}$ and $5 \mathrm{~b}$ show the evolution of the radius of perigee, $R_{P}$, radius of apogee, $R_{a}$, and inclination for the optimal control solution and the state-dependent RL solution respectively. The most noticeable difference is the increase in $R_{a}$ during the transfer. The evolution of the weights can be seen in Fig. 5c again suggesting the emphasis of the eccentricity is slightly reduced compared to the classical case. The average and best time-of-flight values during the learning process are seen in Fig. 5d A batch size of $N=12$ was used, along with a learning rate $\alpha=0.1$ and stochasticity $\sigma=0.03$.

As for dealing with the problem of Q-law chattering, Petropoulos [13] suggests using a very high effectivity threshold in the closing stages of a transfer to help prevent chatter. They also note that relative effectivity might be more suitable during these final stages. We need to investigate this further, but here we demonstrate state-dependent weights trained on a more relaxed convergence criteria are able to improve convergence for stricter tolerances, without considering effectivity thresholds. 


\section{GTO-GEO transfer in Keplerian dynamics with $J_{2}$ and shadow eclipse effects}

Here we consider both time-optimal and mass-optimal transfers for a similar GTO-GEO transfer with a larger $\Delta i$, with $J_{2}$ and eclipse shadow effects (see Appendix $\nabla$ for the implementation). In this case, to match Shannon et al. 2019 [7], the modelled spacecraft has mass $1200 \mathrm{~kg}$, Isp $1800 \mathrm{~s}$, Power $5 \mathrm{~kW}$ and efficiency 0.55 , giving a maximum engine thrust of $0.31 \mathrm{~N}$. The initial and target orbits are shown in Table 5 The convergence criteria are different to the previous section to allow for comparison with the literature.

Table 5 Initial and target orbital elements, and convergence criteria, for a GTO-GEO transfer including $J_{2}$ and eclipse effects

\begin{tabular}{lcccccc}
\hline & $a(\mathrm{~km})$ & $e$ & $i(\mathrm{deg})$ & $\Omega(\mathrm{deg})$ & $\omega(\mathrm{deg})$ & $v(\mathrm{deg})$ \\
\hline \hline Initial & 24,364 & 0.731 & 28.5 & 0.0 & 0.0 & 0.0 \\
Target & $42,165.0$ & 0 & 0 & free & free & free \\
Convergence & 10 & 0.005 & 0.05 & - & - & - \\
\hline
\end{tabular}

As in the Section IV.A, the results for the time- and mass-optimal transfers are shown in Figs 6 and 7 . For both cases, a batch size of $N=24$ was used, with a learning rate $\alpha=0.02$ and stochasticity $\sigma=0.03$. Figure 6 indicates that the eccentricity contribution is reduced in the state-dependent Q-law compared with the classical case. In Fig. 6a it is interesting to note the trajectory reaches the target semi-major axis much earlier than required. This is also reflected in Figs. 6d and 6e, where the contribution of the semi-major axis is minimal for the later part of the transfer. This might allow the control law to avoid the eclipse region and hence increase its thrust time.

For the mass-optimal case, we compare the same transfer to the results presented in [7], fixing the time-of-flight to 120.1 days. Given this time-fixed mass-optimal comparison is similar in time-of-flight to the time-optimal case, it is little surprise that the grey coasting regions seen in Fig $4 \mathrm{~b}$ are quite short and focused at periapsis. Given the constraints imposed by the eclipse periods, the $\left(\mathrm{ToF}_{i+1}^{\text {est }}-\mathrm{ToF}^{\mathrm{aim}}\right)^{2} / \mathrm{ToF}^{\mathrm{aim}}$ term in Eq. 24] was multiplied by $\mathrm{ToF}^{\mathrm{aim}}$ to strengthen this state-dependent contribution and aid convergence.

Table 6 Comparison of state-dependent Q-law performance for GTO-GEO transfer, using a spacecraft with mass $1200 \mathrm{~kg}$, Isp 1800 s and engine thrust $0.31 \mathrm{~N}$ (given Power $5 \mathrm{~kW}$ and efficiency 0.55 ).

\begin{tabular}{llcc}
\hline Objective & Method & Time of Flight (days) & Propellant $(\mathrm{kg})$ \\
\hline \hline Time-of-flight & Shannon et al. 2019 [7] & 119.20 & 170.63 \\
& $\quad(25 \%$ Q-law $+75 \%$ NLP) & & \\
& Q-law $W_{a}, W_{e}=1, W_{i}=5$ & 120.19 & 172.00 \\
& Q-law nominal $W_{\mathcal{X}}=1$ & 136.93 & 196.13 \\
& Q-law PSO fixed $W_{\mathcal{X}}$ & 119.70 & 171.50 \\
& Q-law PSO time-dependent $W_{\mathcal{X}}$ & 119.56 & 171.30 \\
& Q-law AC state-dependent $W_{\mathcal{X}}$ & 119.35 & 170.95 \\
\hline Propellant & Shannon et al. 2019 [7] & 120.1 & 168.1 \\
& $\quad$ (50\% Q-law + 50\% NLP) & & \\
& Q-law PSO fixed $W_{\mathcal{X}}, \eta$ & 120.1 & 170.61 \\
& Q-law PSO time-dependent $W_{\mathcal{X}}, \eta$ & 120.1 & 168.58 \\
& Q-law AC state-dependent $W_{\mathcal{X}}, \eta$ & 120.1 & 169.18
\end{tabular}

The time-of-flight results is within $0.85 \%$ of the orbit averaging result found in [2], and quoted in [7]. In addition, it differs from the $25 \%$ Q-law-75\% NLP result presented in [7] by only $0.1 \%$. There is a 17 day improvement in the time-of-flight compared to the classical Q-law, demonstrating the limitations of user-defined parameters. As for the mass-optimal transfer, we use $1 \mathrm{~kg}$ more than [7], but are better that the fixed PSO results. Given the difficulty the nonlinear programmer (NLP) has when dealing with shadow eclipse effects, this is a promising result. The RL 
process is able to learn the behaviour in the presence of unknown dynamics and perturbations. We hope to extend these capabilities in the future.
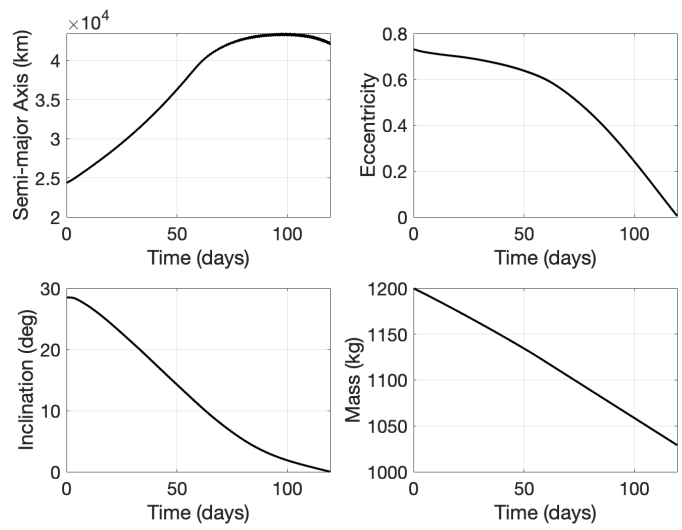

(a) Orbital Parameters

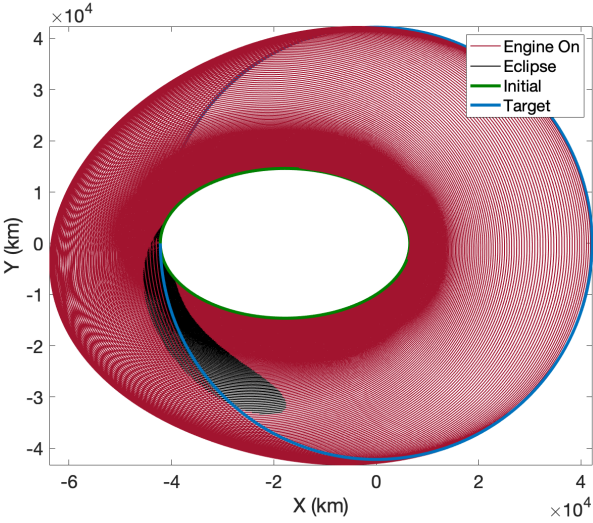

(b) Trajectory

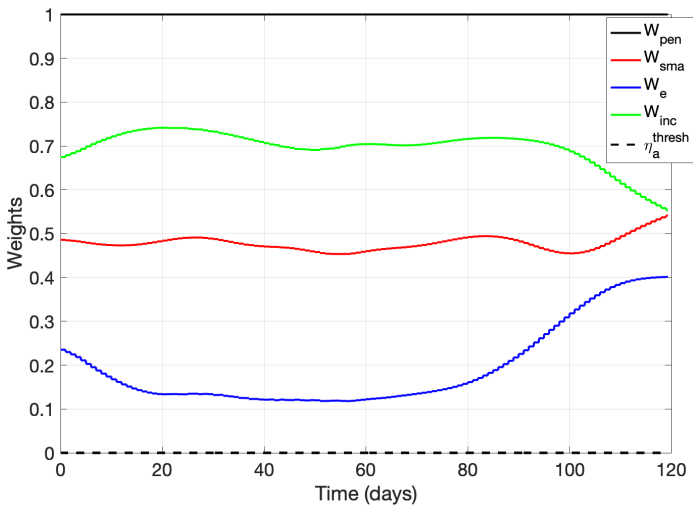

(c) Weights

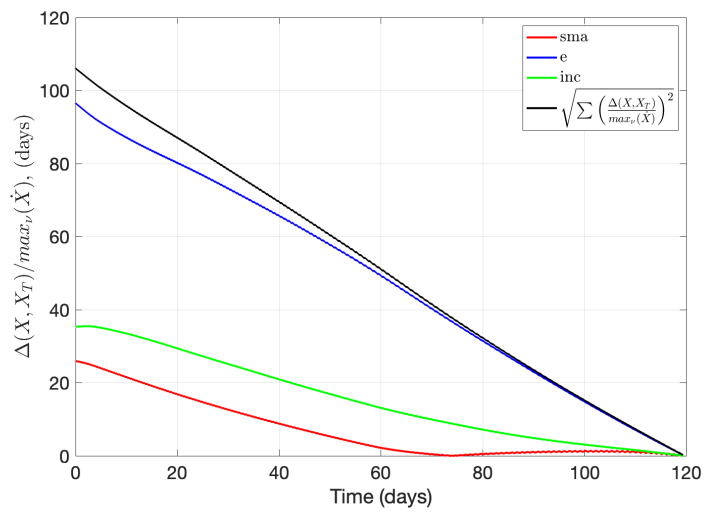

(d) Estimate of the time-to-go from the orbital elements

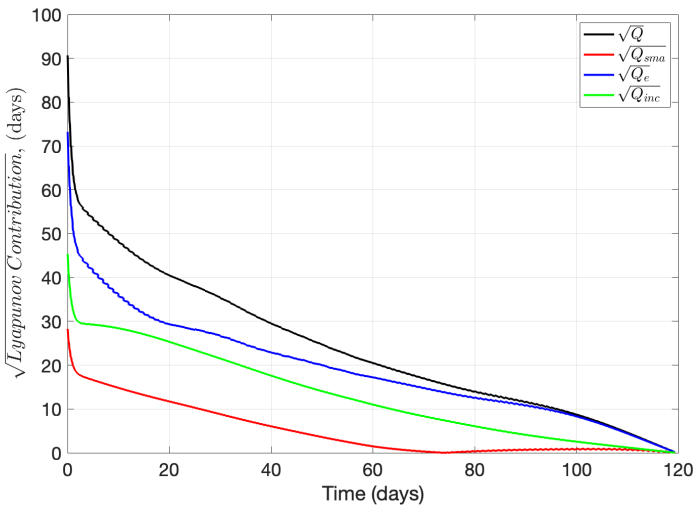

(e) Q-law Evolution

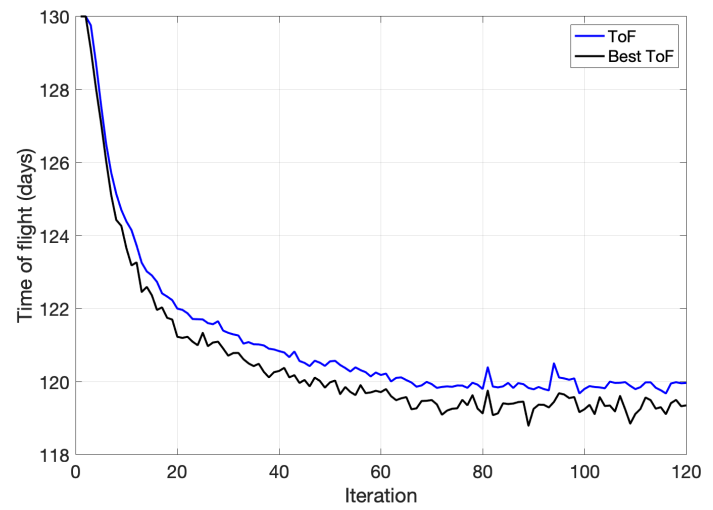

(f) Learning Process

Fig. 6 Time-optimal GTO-GEO transfer using a Q-law with state-dependent weights in Kelperian dynamics with $J_{2}$ and eclipse effects. 

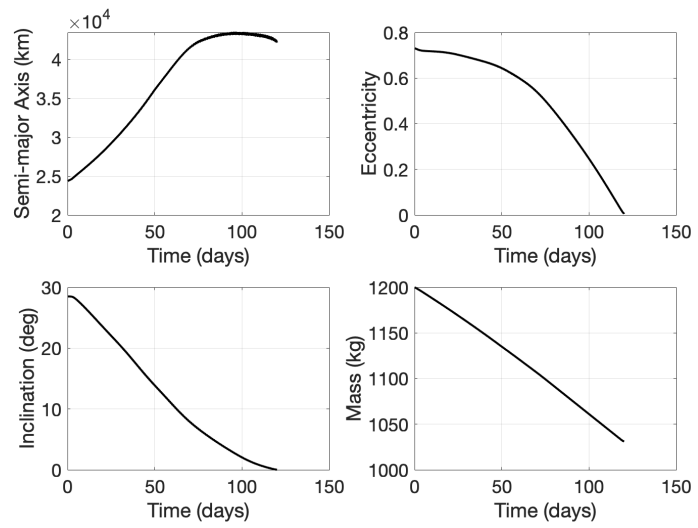

(a) Orbital Parameters

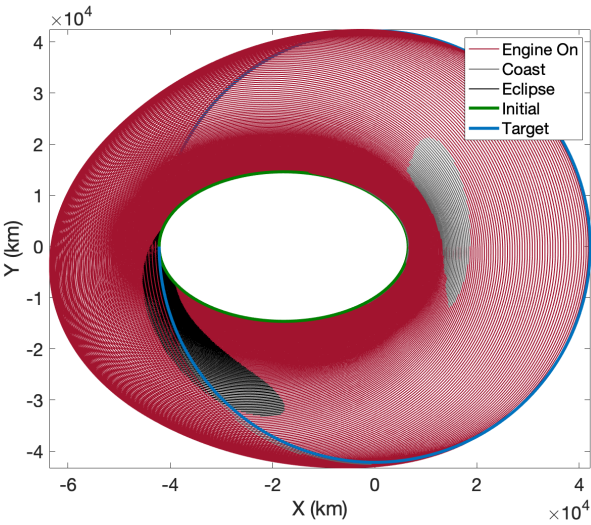

(b) Trajectory

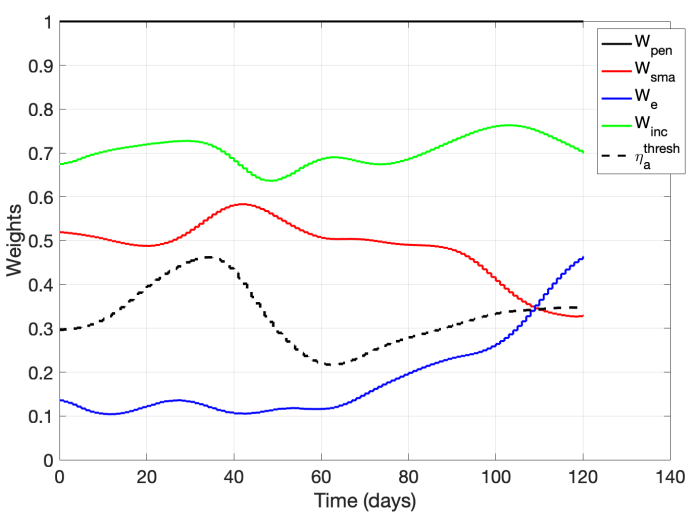

(c) Weights

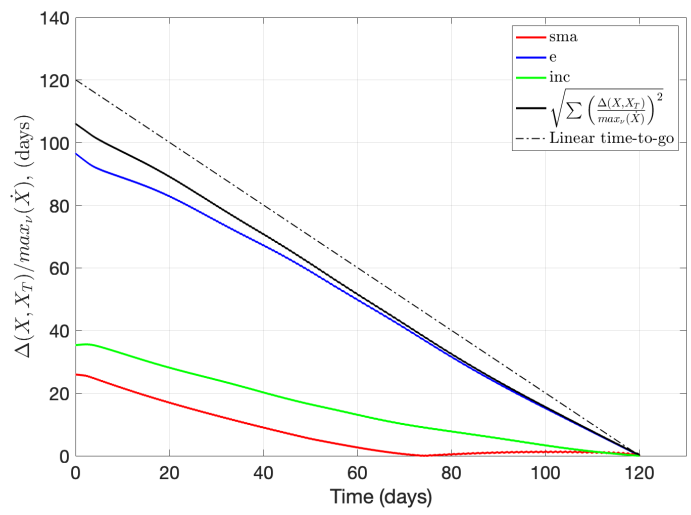

(d) Estimate of the time-to-go from the orbital elements

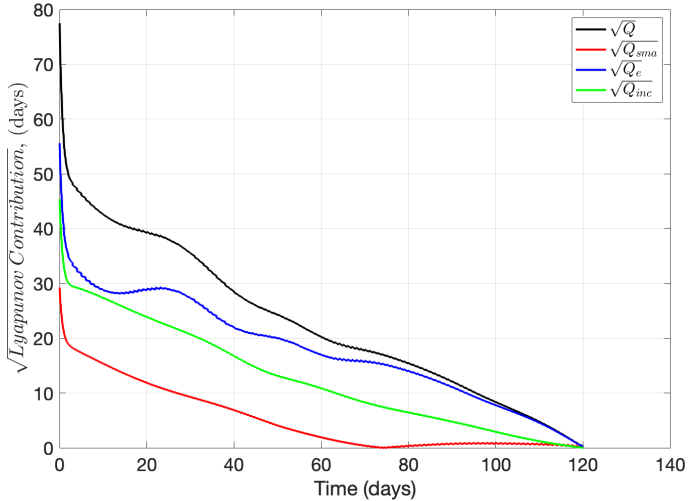

(e) Q-law Evolution

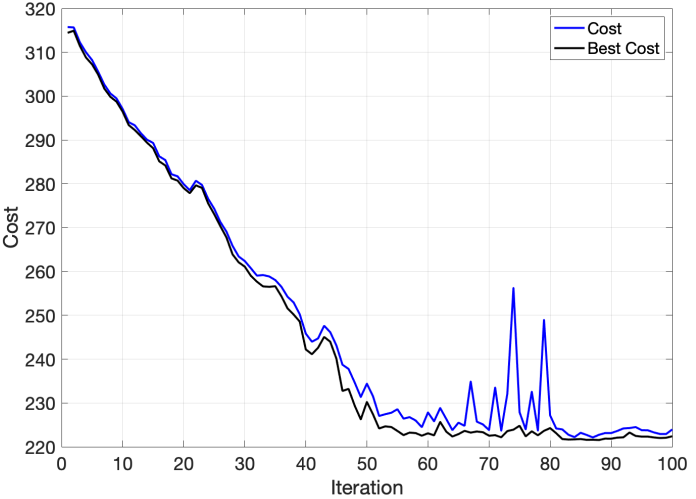

(f) Learning Process

Fig. 7 Mass-optimal GTO-GEO transfer using a Q-law with state-dependent weights and thrust effectivity threshold in Kelperian dynamics with $J_{2}$ and eclipse effects. 


\section{Simulating stability to uncertainties in trajectory}

As a guidance method, there is potential for this approach to be used on-board. In its current format, due to the stochasticity in the learning process, the policy learnt should hold for a distribution of states about the nominal trajectory. One of the concerns when using neural networks is the lack of knowledge when tested on states that lie outside their training area (i.e. when they are extrapolated). However, when combined with the Q-law, a targeting algorithm, much of this stability is retained. This is one of the advantages of using a CLFD control law within the algorithm.

Here we can demonstrate this stability about a nominal trajectory by adding a random displacement to the current state at different points through the transfer. This is done using the state-dependent weights trained for the Keplerian GTO-GEO transfer seen in Section IV.A First, we consider a small deviation in the state, of similar magnitude to the stochastic variations during training. The disturbance magnitudes were $\sim 1 \mathrm{~km}$ for the semi-major axis, $\sim 0.001$ for the eccentricity and $\sim 0.01$ degrees for the inclination, RAAN and argument of periapsis. Figure 8 shows the results of 50 simulations when the disturbance is added after 0, 25, 50, 75, 100 and 125 days. The red line indicated the time-of-flight for the undisturbed solution, 138.35 days. For each simulation, we can calculate its expected time-to-go immediately after the disturbance using the Q-law formulation. This allows us to compare the expected time-to-go for the disturbed trajectory with the undisturbed trajectory, and helps indicate whether the disturbance was detrimental or advantageous in terms of time-of-flight. The difference between the two is shown in the colourbar.

We can see the algorithm is able to handle these deviations comfortably and still converge in a good time-of-flight, comparable or better than the expected time-of-flight. In addition, the resulting effect of a disturbance is greater earlier in the transfer. The reason for this behaviour is not yet fully understood. One possible explanation may reside in the increased state-space exploration later in the transfer, when the stochastic variations during a training trajectory have accumulated a greater deviation from the nominal trajectory. Hence, the learnt policy might be better equipped to handle these later in the transfer. In addition, as the perturbations are similar in magnitude to the convergence criteria, it is possible a disturbance later in the trajectory is partially absorbed by the flexibility allowed by the convergence criteria. However, further analysis is required to determine this.
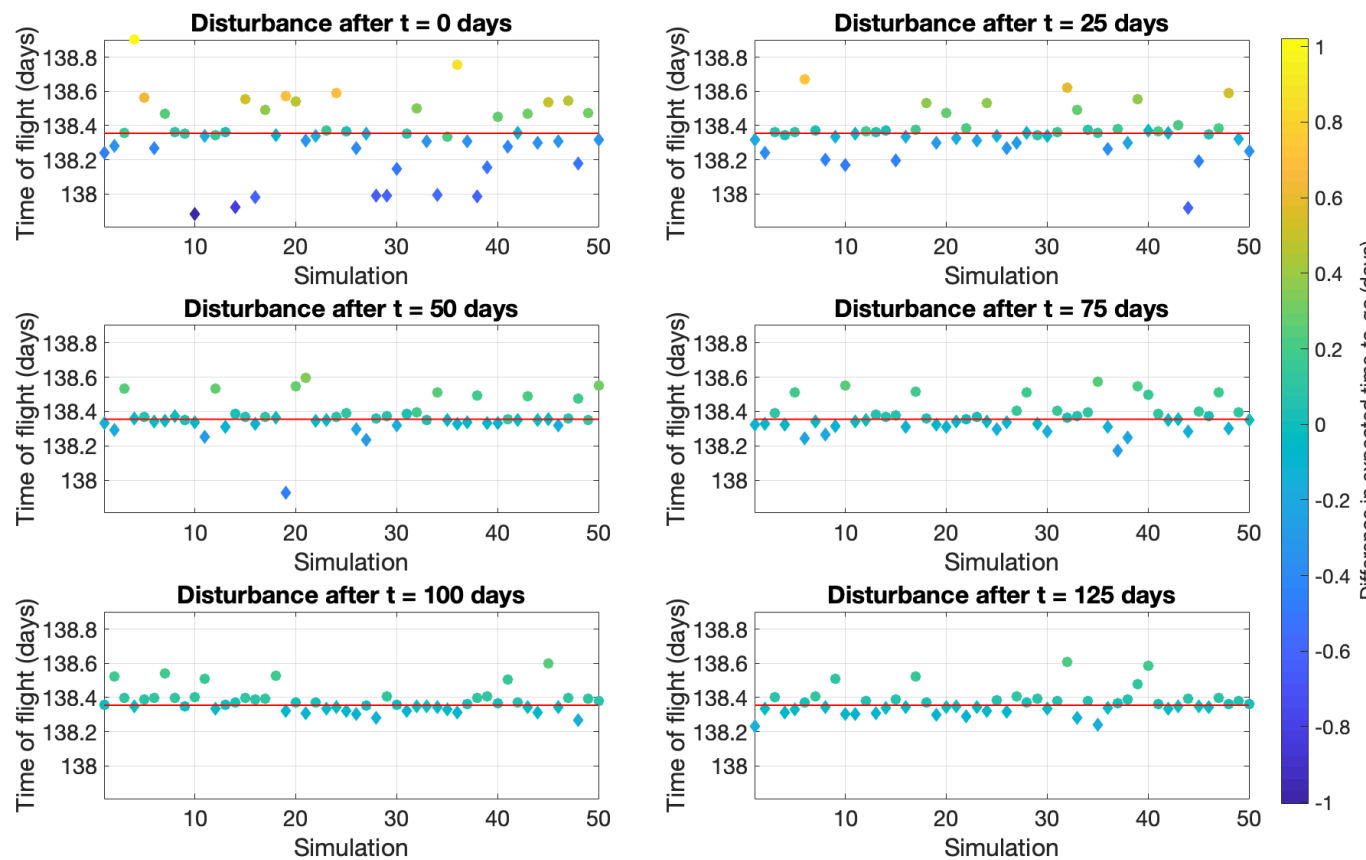

Fig. 8 Time-optimal GTO-GEO transfer performance with disturbances in the trajectory at different stages throughout the transfer (RMS $a \sim 1 \mathbf{~ k m}, e \sim 0.001, i \sim 0.01 \mathbf{d e g}$ ). The red line indicates the undisturbed solution with a time-of-flight of 138.35 days. The colourbar indicates the difference between the estimated time-to-go for the disturbed and undisturbed trajectory at the time of disturbance. 
We also investigate the stability to much larger errors about the nominal trajectory, larger than the size of the stochasticity during training. In Fig. 9 the disturbance magnitudes are $\sim 10 \mathrm{~km}$ for the semi-major axis, $\sim 0.01$ for the eccentricity and $\sim 0.1$ degrees for the inclination, RAAN and argument of periapsis. Again, the algorithm is able to handle these disturbances with ease, and the trajectories are all able to converge. In all cases, the resulting time-of-flight remains below the classical Q-law value for the same transfer.
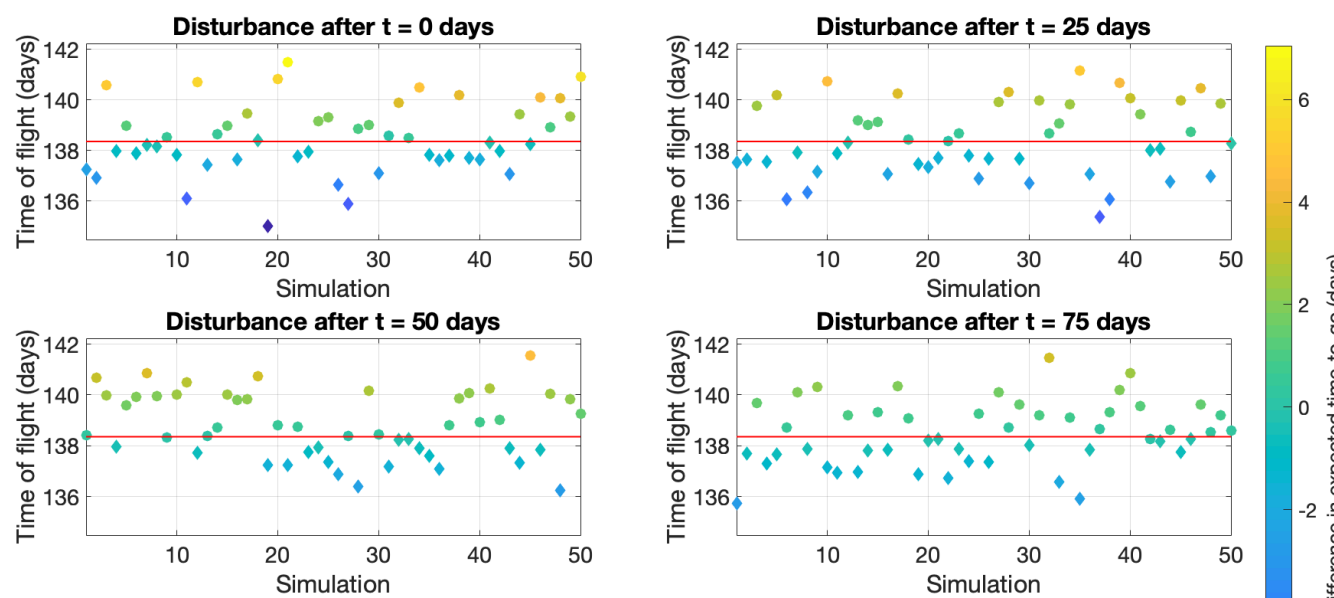

Disturbance after $t=100$ days

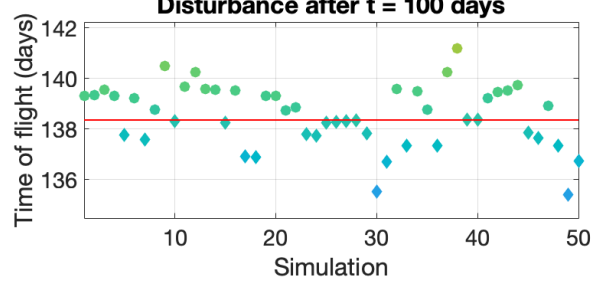

Disturbance after $t=125$ days

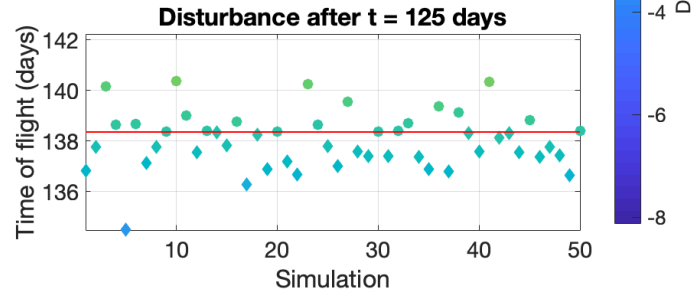

Fig. 9 Time-optimal GTO-GEO transfer performance with disturbances in the trajectory at different stages throughout the transfer (RMS $a \sim 10 \mathbf{~ k m}, e \sim 0.01, i \sim 0.1 \mathbf{~ d e g}$ ). The red line indicates the undisturbed solution with a time-of-flight of 138.35 days. The colourbar indicates the difference between the estimated time-to-go for the disturbed and undisturbed trajectory at the time of disturbance.

\section{Conclusion}

In this work we have presented a novel approach to making the parameters of the Lyapunov-based Q-law statedependent. This is achieved using a single-layer actor-critic reinforcement learning approach, inspired by similar approaches in spacecraft landing and relative motion guidance problems. The results were simulated for GTO-GEO transfers, first in Keplerian dynamics, and then including eclipse and $J_{2}$ perturbation effects as a proof of concept. For both time- and mass-optimal transfers, the results significantly improve on the classical Q-law performance, demonstrating state-dependent weights improve the optimality of closed-loop feedback-driven (CLFD) control laws, and are comparable with similar techniques and optimal control solutions found in the literature, validating the performance.

The advantage of combining a CLFD control law with a reinforcement learning architecture is seen when studying the stability to disturbances about a nominal trajectory. Adding the Q-law to an actor-critic agent increases the stability of the agent, particularly outside the training space. This inbuilt stability to small variations provides a robust nature to the control law and paves the way for future on-board use. In future work we will extend the ideas presented here to higher-fidelity dynamics, incorporating the effects of perturbations in the work. In addition, we hope to investigate and compare the use of multi-layer neural networks with the single-layer implementation presented here. Reinforcement learning algorithms are well suited to unknown environments and this is a major motivation for this line of research. 


\section{Appendix}

\section{Eclipse Implementation}

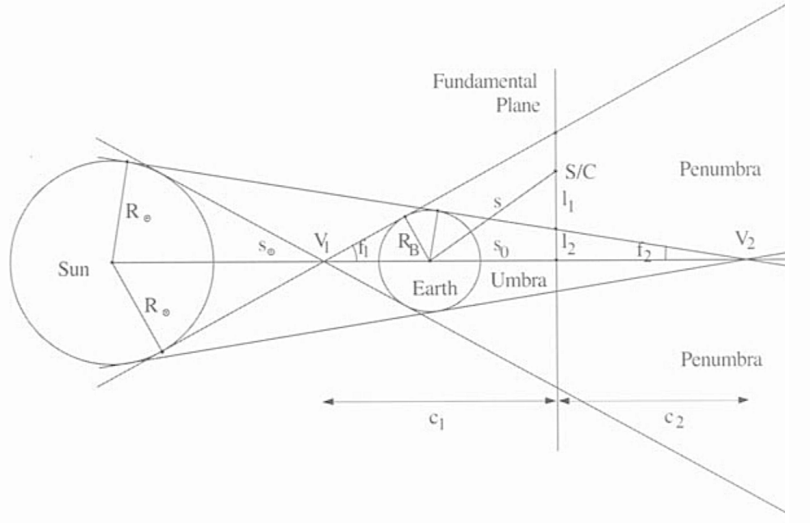

(a)

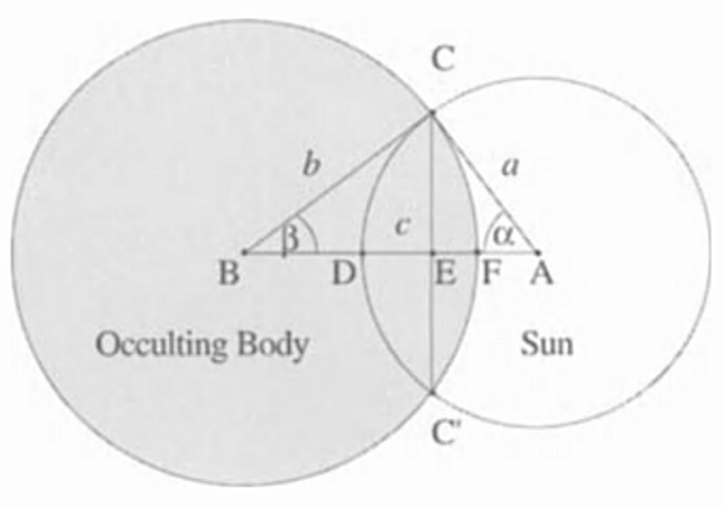

(b)

Fig. 10 Eclipse geometry. The spacecraft is indicated as S/C. Taken from Montenbruck and Gill 2005 [41]

Incorporating eclipse effects requires knowledge of the positions of the spacecraft, Earth and Sun. The Sun's ephemeris is thus obtained using NAIF's SPICE Toolkit [42]. This can give the position of the Sun as an $x y z$ coordinate in the Earth-centred inertial reference frame (ECI). A spacecraft's state in COE can also be converted to $x y z$ coordinates. Figure 10 shows the geometry assumed in this work: both the Earth and Sun are approximated as occulting discs. Assuming this set-up, full and partial eclipses can be modelled. The thrust of the spacecraft is modified by the eclipse parameter $\eta_{\text {eclipse }}$ :

$$
\eta_{\text {eclipse }}=1-\frac{\text { Area Occulted }}{\text { Total Area }}=1-\frac{A}{\pi R_{\odot \text { app }}^{2}}
$$

The occulted area may be expressed as

$$
\begin{gathered}
A=2\left(A_{\mathrm{BCF}}-A_{\mathrm{BCE}}\right)+2\left(A_{\mathrm{ACD}}-A_{\mathrm{ACE}}\right) \\
=a^{2} \arccos (x / a)+b^{2} \arccos ((c-x) / b)-c y .
\end{gathered}
$$

\section{$J_{2}$ Implementation}

The gravitational perturbation due to Earth's oblateness, $J_{2}$, causes a short term variation of all orbital parameters, and a secular variation of $\Omega$ and $\omega$, given by Eqs. [27) [10]. These are the average variation over one orbit.

$$
\dot{\Omega}_{\mathrm{av}}=-\frac{3}{2} \tilde{n} J_{2}\left(\frac{R_{E}}{p}\right)^{2} \cos i \quad \dot{\omega}_{\mathrm{av}}=+\frac{3}{4} \tilde{n} J_{2}\left(\frac{R_{E}}{p}\right)^{2}\left(5 \cos ^{2} i-1\right)
$$

Here, $J_{2}=1.082635854 \times 10^{-3}, R_{E}$ is the mean Earth radius (reference geoid) $6378.14 \mathrm{~km}$ and $\tilde{n}^{2} a^{3}=\mu$. We can incorporate these variations into Gauss's Variational Equations by converting to the spacecraft's $R T N$ frame.

$$
\boldsymbol{a}_{J_{2}}=\frac{-3 \mu J_{2} R_{E}^{2}}{r^{4}}\left[\left(\frac{1}{2}-\frac{3 \sin ^{2} i \sin ^{2}(\omega+v)}{2}\right) \hat{\boldsymbol{e}}_{R}+\sin ^{2} i \sin (\omega+v) \cos (\omega+v) \hat{\boldsymbol{e}}_{T}+\sin i \cos i \sin (\omega+v) \hat{\boldsymbol{e}}_{N}\right]
$$

\section{Acknowledgments}

Harry Holt is funded by Surrey Satellite Technology Limited (SSTL). HH thanks Andrea Turconi, Yoshi Hashida and Steve Eckersley from SSTL and Chris Bridges from Surrey Space Centre (SSC) for their input to the project. HH also thanks Nicola Baresi from SSC for his advice and input to the project, and his useful comments on the overall structure of the work. 


\section{References}

[1] Betts, J. T., Practical Methods for Optimal Control and Estimation Using Nonlinear Programming, Society for Industrial and Applied Mathematics, 2010.

[2] Conway, B. A., Spacecraft trajectory optimization, Cambridge University Press, 2010.

[3] Shirazi, A., Ceberio, J., and Lozano, J. A., "Spacecraft trajectory optimization: A review of models, objectives, approaches and solutions," Progress in Aerospace Sciences, Vol. 102, No. August, 2018, pp. 76-98.

[4] Kluever, C. A., "Simple guidance scheme for low-thrust orbit transfers," Journal of Guidance, Control, and Dynamics, Vol. 21, No. 6, 1998, pp. 1015-1017.

[5] Petropoulos, A. E., "Simple control laws for low-thrust orbit transfers," AAS/AIAA Astrodynamics Specialists Conference, 2003.

[6] Gao, Y., and Li, X., "Optimization of low-thrust many-revolution transfers and Lyapunov-based guidance," Acta Astronautica, Vol. 66, No. 1-2, 2010, pp. 117-129.

[7] Shannon, J. L., Ozimek, M. T., Atchison, J. A., and Christine, M., "Q-law aided Direct Trajectory Optimization for the High-fidelity, Many-revolution Low-thrust Orbit Transfer Problem,” AAS, 2019, p. 448.

[8] Lee, S., Petropoulos, A., and von Allmen, P., "Low-thrust Orbit Transfer Optimization with Refined Q-law and Multi-objective Genetic Algorithm," Advances In The Astronautical Sciences, 2005.

[9] Varga, G., and Sánchez Pérez, J. M., "Many-Revolution Low-Thrust Orbit Transfer Computation Using Equinoctial Q-Law Including J2 and Eclipse Effects,” Icatt 2016, 2016, pp. 2463-2481.

[10] Schaub, H., and Junkins, J. L., Analytical Mechanics of Space Systems, Fourth Edition, American Institute of Aeronautics and Astronautics, Inc., 2018.

[11] Joseph, B. E., "Lyapunov Feedback Control in Equinoctial Elements Applied to Low Thrust Control of Elliptical Orbit Constellations," Msc thesis, Massachusetts Institute of Technology, 2006.

[12] Naasz, B. J., "Classical Element Feedback Control for Spacecraft Orbital Maneuvers," Msc thesis, Virginia Polytechnic Institute and State University, 2002.

[13] Petropoulos, A. E., "Refinements to the Q-law for low-thrust orbit transfers," Advances in the Astronautical Sciences, Vol. 120, 2005, pp. 963-982.

[14] Hatten, N., “A Critical Evaluation of Modern Low-Thrust, Feedback-Driven Spacecraft Control Laws," Master's thesis, The University of Texas at Austin, 2012.

[15] Ruggiero, A., Pergola, P., Marcuccio, S., Andrenucci, M., Manager, P., and President, V., "Low-Thrust Maneuvers for the Efficient Correction of Orbital Elements," 32nd International Electric Propulsion Conference, 2011, pp. 1-13.

[16] Falck, R., Sjauw, W., and Smith, D., "Comparison of Low-Thrust control laws for application in planetocentric space," 50th AIAA/ASME/SAE/ASEE Joint Propulsion Conference 2014, 2014, pp. 1-14.

[17] Maddock, C., and Vasile, M., "Extension of the proximity-quotient control law for low-thrust propulsion," International Astronautical Federation - 59th International Astronautical Congress 2008, IAC 2008, Vol. 8, No. July, 2008, pp. 4901-4915.

[18] Baresi, N., Dell'Elce, L., Cardoso dos Santos, J., and Kawakatsu, Y., “Orbit maintenance of quasi-satellite trajectories via mean relative orbit elements," Iac-2018, 2018, pp. 1-11.

[19] Epenoy, R., and Pérez-Palau, D., "Lyapunov-based low-energy low-thrust transfers to the Moon," Acta Astronautica, Vol. 162, No. June, 2019, pp. 87-97.

[20] Izzo, D., Märtens, M., and Pan, B., "A Survey on Artificial Intelligence Trends in Spacecraft Guidance Dynamics and Control," arXiv preprint arXiv:1812.02948, 2018.

[21] Yang, D. L., Xu, B., and Zhang, L., "Optimal low-thrust spiral trajectories using Lyapunov-based guidance,” Acta Astronautica, Vol. 126, 2016, pp. 275-285.

[22] Gaudet, B., Linares, R., and Furfaro, R., "Deep Reinforcement Learning for Six Degree-of-Freedom Planetary Powered Descent and Landing," arXiv preprint arXiv:1810.08719, 2018. 
[23] Gaudet, B., and Furfaro, R., "Robust Spacecraft Hovering Near Small Bodies in Environments with Unknown Dynamics Using Reinforcement Learning," AIAA/AAS Astrodynamics Specialist Conference, , No. May 2018, 2012.

[24] Miller, D., and Linares, R., “Low-Thrust Optimal Control Via Reinforcement Learning,” AAS, 2019, pp. 1-20.

[25] Scorsoglio, A., “Adaptive ZEM/ZEV feedback guidance for rendezvous in lunar NRO with collision avoidance,” Master's thesis, Politecnico Di Milano, University of Arizona, 2018.

[26] Scorsoglio, A., Furfaro, R., Linares, R., and Massari, M., "Actor-critic reinforcement learning approach to relative motion guidance in near-rectilinear orbit,” Advances in the Astronautical Sciences, 2019, pp. Paper AAS-19-441 pp. 1-20.

[27] Scorsoglio, A., and Furfaro, R., "ELM-based Actor-Critic Approach to Lyapunov Vector Fields Relative Motion Guidance in Near-Rectilinear Orbit,” 2019 AAS/AIAA Astrodynamics Specialists Conference, 2019, pp. Paper AAS-19-692 pp. 1-20.

[28] Harris, A., Teil, T., and Schaub, H., "Spacecraft Decision-making Autonomy using Deep Reinforcement Learning," AAS, 2019, pp. $1-19$.

[29] Bianchi, R. A. C., Ribeiro, C. H. C., and Costa, A. H. R., "On the Relation Between Ant Colony Optimization and Heuristically Accelerated Reinforcement Learning," 1st International Workshop on Hybrid Control of Autonomous System, , No. Hycas, 2009, pp. 49-55.

[30] Furfaro, R., Scorsoglio, A., Linares, R., and Massari, M., “Adaptive Generalized ZEM-ZEV Feedback Guidance for Planetary Landing via a Deep Reinforcement Learning Approach,” Acta Astronautica (In Review), 2020.

[31] Battin, R. H., An Introduction to the Mathematics and Methods of Astrodynamcis, AIAA Education Series, New York, 1987.

[32] Sutton, R., and Barto, A., Reinforcment Learning: An Introduction, MIT Press, Cambridge, 1998.

[33] Grondman, I., Busoniu, L., Lopes, G., and Babuska, R., “A survey of actor-critic reinforcement learning,” IEEE Transactions on Systems, Man, and Cybernetics, Part C: Applications and Reviews, Vol. 42, No. 6, 2012, pp. 1291-1307.

[34] Sutton, R., McAllester, D., Singh, S., and Mansour, Y., "Policy Gradient Methods for Reinforcement Learning with Function Approximation,” Advances in Neural Information Processing Systems 12, 1999, pp. pages 1057-1063.

[35] Silver, D., Lever, G., Hess, N., Degris, T., Wierstra, D., and Riedmiller, M., "Deterministic Policy Gradient (DPG)," ICML, 2014.

[36] Williams, R. J., "Simple statistical gradient-following algorithms for connectionist reinforcement learning," Machine Learning, Vol. 8, No. 3-4, 1992, pp. 229-256.

[37] Huang, G. B., "What are Extreme Learning Machines? Filling the Gap Between Frank Rosenblatt's Dream and John von Neumann's Puzzle," Cognitive Computation, Vol. 7, No. 3, 2015, pp. 263-278.

[38] Kingma, D. P., and Ba, J., “Adam: A Method for Stochastic Optimization,” ICLR, 2015, pp. 1-15.

[39] Geffroy, S., and Epenoy, R., "Optimal low-thrust transfers with constraints - Generalization of averaging techniques," Acta Astronautica, Vol. 41, No. 3, 1997, pp. 133-149.

[40] Fasano, G., and Pinter, J. (eds.), Modeling and Optimization in Space Engineering: State of the Art and New Challenges, Springer, 2013.

[41] Montenbruck, O., and Gill, E., Satellite Orbits: Models, Methods and Applications, Springer, 2005.

[42] NAIF, “SPICE: An Observation Geometry System for Planetary Science Missions,”, 2017. 